\title{
Asymptotic behaviour of iterated piecewise monotone maps
}

\author{
JÜRGEN WILLMS \\ Universität Bielefeld, Fakultät für Mathematik, 4800 Bielefeld 1, West Germany
}

(Received 22 November 1986)

Abstract. In this paper the asymptotic behaviour of piecewise monotone functions $f: I \rightarrow I$ with a finite number of discontinuities is studied (where $I \subseteq \mathbb{R}$ is a compact interval). It is shown that there is a finite number of $f$-almost-invariant subsets $C_{1}, \ldots, C_{r}, R_{1}, \ldots, R_{s}$, where each $C_{i}$ is a disjoint union of closed intervals and each $R_{j}$ is a Cantor-like subset of $I$, such that if $x$ is a 'typical' point in $I$ (in a topological sense) then exactly one of the following three possibilities will happen:

(1) $\left\{f^{n}(x)\right\}_{n \geq 0}$ eventually ends up in some $C_{i}$.

(2) $\left\{f^{n}(x)\right\}_{n \geq 0}$ is attracted to some $R_{j}$.

(3) $\left\{f^{n}(x): n \geq 0\right\}$ is contained in an open, invariant set $Z \subseteq I$, which is such that for each $n \geq 1 f^{n}$ is monotone and continuous on each connected component of $Z$.

Moreover, $f$ acts topologically transitively on each $C_{i}$ and minimally on each $R_{j}$. Furthermore, it is shown how the sets $C_{1}, \ldots, C_{r}, R_{1}, \ldots, R_{s}$ can be constructed. Finally, our results are applied to some examples.

\section{Introduction}

In the last few years there has been considerable interest in the qualitative behaviour of iterates of maps on an interval into itself (see, for instance, [2], [6], [9], [11], $[12],[14]$ ). Although they are the simplest examples of non-linear (discrete) dynamical systems their asymptotic behaviour can exhibit a surprisingly complex structure. One-dimensional maps have been used as models for various systems (see, for instance, the models of density dependent population growth studied in [10]). It is known that in certain cases the asymptotic behaviour of higher-dimensional (discrete as well as continuous) dynamical systems can be, at least partly, described by iterates of maps on an interval into itself. For instance, in [19] the Lorenz attractor is described as the inverse limit of a semi-flow on a two-dimensional branched manifold. The Poincaré map of this semi-flow is a function on a bounded interval $[a, b]$ into itself which has a single discontinuity at $c=(a+b) / 2$ and is strictly increasing on $[a, c)$ and $(c, b]$. Interval exchange transformations, Newton's method for determining the zeros of a polynomial (identifying $\overline{\mathbb{R}}$ with the unit interval) and the $\beta$-transformations discussed in [17] are some more examples for discrete dynamical systems on a compact interval, having a finite number of discontinuities.

In [15] Preston studied the asymptotic behaviour of iterates of piecewise monotone continuous functions on a compact interval $I$ into itself, i.e. continuous functions 
$f: I \rightarrow I$ with only a finite number of points at which $f$ is not strictly monotone (an improved and much simplified version of [15] is contained in $\S \S 2,3$ and 4 of [16]). The present work generalizes his main result in that a finite number of discontinuities is allowed. More exactly, let $a, b \in \mathbb{R}$ with $a<b$ and let $I$ be the closed interval with endpoints $a$ and $b$. For technical reasons we consider a point $\omega$ not contained in $\mathbb{R}$ and put $I^{\prime}=I \cup\{\omega\}$. We call a map $f: I^{\prime} \rightarrow I^{\prime}$ piecewise monotone on $I$ if there exist $m \geq 1$ and $a=d_{0}<d_{1}<\cdots<d_{m}=b$ such that $f^{-1}(\{\omega\})=\left\{d_{0}, d_{1}, \ldots, d_{m}, \omega\right\}$ and, for all $0 \leq k \leq m-1, f$ is continuous and strictly monotone on each of the intervals $\left(d_{k}, d_{k+1}\right) . \mathcal{N}(I)$ will denote the set of piecewise monotone maps on $I$. For $f \in \mathcal{N}(I)$ put $S(f)=f^{-1}(\{\omega\}) \cap I$. Now let $f \in \mathcal{N}(I)$ be fixed. We define $f^{n} \in \mathcal{N}(I)$, the nth. iterate of $f$, inductively by $f^{0}(x)=x$ and $f^{n+1}(x)=f\left(f^{n}(x)\right)$ for all $x \in I^{\prime}$ and $n \geq 0$. Unlike in [7] where the structure of the non-wandering set is studied (using symbolic dynamics) our aim is to analyse the asymptotic behaviour of $\left\{f^{n}(x)\right\}_{n \geq 0}$ for a 'typical' point $x$ of $I$ (in a topological sense).

After some preliminaries in $\S 2$ we study in $\S 3$ some basic properties of sinks and homtervals of $f$. $\S 4$ is concerned with some elementary properties of topologically transitive $f$-cycles and $f$-register-shifts. The main result is stated and proved in $\S 5$. It says that there are only a finite number of topologically transitive $f$-cycles $C_{1}, \ldots, C_{r}$ and $f$-register-shifts $R_{1}, \ldots, R_{s}$ and that for all points $x$ lying in some residual subset of $I$ exactly one of the following three things will happen:

(1) $\left\{f^{n}(x)\right\}_{n \geq 0}$ eventually ends up in some topologically transitive $f$-cycle $C_{i}$.

(2) $\left\{f^{n}(x)\right\}_{n \geq 0}$ is attracted to some $f$-register-shift $\boldsymbol{R}_{j}$.

(3) $\left\{f^{n}(x)\right\}_{n \geq 0}$ eventually ends up in some sink or some homterval of $f$ (in particular, $\left\{f^{n}(x): n \geq 0\right\}$ is contained in an open, invariant set $Z \subseteq I$, which is such that for each $n \geq 1, f^{n}$ is monotone and continuous on each connected component of $Z$ ).

In $\S 6$ we study some more properties of $f$-register-shifts and topologically transitive $f$-cycles. In particular, we show that each $f$-register-shift $R$ is a Cantor-like set, that $R-Q \subseteq f(R-S(f)) \subseteq R$ where $Q$ is a finite subset of $I$ and that for each $x \in R$ either $\left\{f^{n}(x): n \geq 0\right\}$ contains a singular point of $f$, i.e. an element of $S(f)$, or $\left\{f^{n}(x): n \geq 0\right\}$ is dense in $R$. Moreover, we prove that each topologically transitive $f$-cycle is in fact strongly transitive. Finally, in $\S 7$ we apply our results to some examples.

Acknowledgement. I would like to thank Professor Chris Preston for his helpful suggestions and comments, and in particular for his improvements to the clarity of the definition of register-shifts. I am also grateful to the referee for his remarks and recommendations.

This paper is a shortened and revised version of the author's Dissertation (Bielefeld 1985) with the same title.

\section{Piecewise monotone functions}

Let $a, b \in \mathbb{R}$ with $a<b$ and let $\omega$ be some point not contained in $\mathbb{R}$. Put $I=[a, b]$ and $I^{\prime}=I \cup\{\omega\}$; we consider $I^{\prime}$ as a topological space by calling a subset $A$ of $I^{\prime}$ open if there exists an open subset $U$ of $\mathbb{R}$ such that $U \cap I=A \cap I$. 
A function $f: I^{\prime} \rightarrow I^{\prime}$ is called piecewise monotone on $I$ if there exists $m \geq 1$ and $a=d_{0}<d_{1}<\cdots<d_{m}=b$ such that

(2.1) $f$ is continuous and strictly monotone on each of the open intervals $\left(d_{k}, d_{k+1}\right)$, $k=0,1, \ldots, m-1$, and

(2.2) $f^{-1}(\{\omega\})=\left\{d_{0}, d_{1}, \ldots, d_{m}, \omega\right\}$.

$\mathcal{N}(I)$ will denote the set of piecewise monotone functions on $I$. Throughout this paper we assume that $f$ is a fixed element of $\mathcal{N}(I)$.

For $n \geq 0$ define $f^{n}: I^{\prime} \rightarrow I^{\prime}$ inductively by $f^{0}(x)=x$ and $f^{n+1}(x)=f\left(f^{n}(x)\right)$ for all $x \in I^{\prime}$. It is easy to check that the composition of two elements of $\mathcal{N}(I)$ is again an element of $\mathcal{N}(I)$; hence we have $f^{n} \in \mathcal{N}(I)$ for each $n \geq 1$. Let $S(f)$ denote the set of singular points of $f$ in $I$, i.e. $S(f)=f^{-1}(\{\omega\}) \cap I$. Put $S\left(f^{0}\right)=\varnothing$; it is not difficult to show that for all $n, k \geq 0$ and $A \subseteq I$ we have

$$
\begin{aligned}
S\left(f^{n}\right) & =\left\{x \in I: f^{j}(x) \in S(f) \text { for some } 0 \leq j<n\right\} \\
& =\left\{x \in I: f^{n}(x)=\omega\right\}, \\
f^{n}\left(A-S\left(f^{n}\right)\right) & =f^{n}(A) \cap I
\end{aligned}
$$

and

$$
f^{n}\left(f^{k}\left(A-S\left(f^{k}\right)\right)-S\left(f^{n}\right)\right)=f^{n+k}\left(A-S\left(f^{n+k}\right)\right) .
$$

Let $n \geq 1$; note that $f^{n}$ is continuous and monotone on an open interval $J \subseteq I$ if and only if $J \cap S\left(f^{n}\right)=\varnothing$ and that $J \cap S\left(f^{n}\right)=\varnothing$ if and only if $f^{n}(J) \subset I$. Note also that if $f^{n}(x) \in I$ for some $x \in I$ then there exists $\varepsilon>0$ such that $f^{n}$ is continuous and monotone on $(x-\varepsilon, x+\varepsilon)$; in particular, $f^{n}\left(A-S\left(f^{n}\right)\right)$ and $f^{-n}(A)$ are open whenever $A$ is an open subset of $I$ (where $f^{-n}(A)=\left\{x \in I: f^{n}(x) \in A\right\}$ ). Moreover, if $A$ is an open interval contained in $I$ then $f^{n}\left(A-S\left(f^{n}\right)\right)$ is a finite union of open intervals.

A subset $A \subseteq I$ is called $f$-almost-invariant if $f(A-S(f)) \subseteq A$. The union and the intersection of any number of $f$-almost-invariant subsets of $I$ are $f$-almost-invariant. Furthermore, it is easy to show that if $A \subseteq I$ is $f$-almost-invariant then int $(A)$ and $\bar{A}$ are both $f$-almost-invariant and that for each $n \geq 1, A$ is also $f^{n}$-almost-invariant.

We call $A \subseteq I$-biinvariant if $A$ is $f$-almost-invariant and $f^{-1}(A) \subseteq A$. Again the union and the intersection of $f$-biinvariant subsets of $I$ are $f$-biinvariant.

\section{Sinks and homtervals}

All results and proofs in this section are almost identical with the ones in [16]. For the convenience of the reader we give the proofs below.

A non-empty, open interval $J \subseteq I$ is called a sink of $f$ if there exists $m \geq 1$ such that $f^{m}(J) \subseteq J$. Note that if $J$ is a sink of $f$ then $f^{n}(J) \cap S(f)=\varnothing$ and hence $J \cap S\left(f^{n}\right)=\varnothing$ for all $n \geq 0$; thus for each $n \geq 0, f^{n}$ is continuous and monotone on $J$.

LEMMA 3.1 (cf. [16, lemma 4.1]). Let $U \subseteq I$ be a non-empty open interval such that $f^{n}(U) \subseteq I$ for each $n \geq 0$. If $U \cap f^{m}(U) \neq \varnothing$ for some $m \geq 1$ then $U$ is contained in a $\operatorname{sink}$ of $f$. 
Proof. Put $J=\bigcup_{k \geq 0} f^{k m}(U)$; then $U \subseteq J \subseteq I$ and $J$ is a non-empty open interval with $f^{m}(J) \subseteq J$.

We call a non-empty open interval $L \subseteq I$ a homterval of $f$ if for each $n \geq 0$ we have $f^{n}(L) \subseteq I$ and $f^{n}(L)$ is not contained in any sink of $f$. Note that if $L$ is a homterval of $f$ then $f^{n}(L) \cap S(f)=\varnothing$ and hence $L \cap S\left(f^{n}\right)=\varnothing$ for all $n \geq 0$; thus $f^{n}$ is continuous and monotone on $L$ for each $n \geq 0$. We emphasize that by proposition 3.3 (3) our definition of a homterval is equivalent to the usual one (see for instance [5]). For the construction of functions having homtervals see for example [5] or [16].

Proposition 3.2 (cf. [16, proposition 4.3]). (1) If $J_{1}, J_{2}$ are sinks (resp. homtervals) of $f$ with $J_{1} \cup J_{2} \neq \varnothing$ then $J_{1} \cup J_{2}$ is also a sink (resp. homterval) of $f$.

(2) If $J$ is a sink (resp. homterval) of $f$ then for each $n \geq 0, f^{n}(J)$ is also a sink (resp. homterval) of $f$.

(3) Each sink of $f$ is contained in a maximal sink of $f$; if $J_{1}$ and $J_{2}$ are maximal sinks of $f$ then either $J_{1}=J_{2}$ or $J_{1} \cap J_{2}=\varnothing$.

(4) Each homterval of $f$ is contained in a maximal homterval of $f$; if $L_{1}$ and $L_{2}$ are maximal homtervals of $f$ then either $L_{1}=L_{2}$ or $L_{1} \cap L_{2}=\varnothing$.

Proof. (1) and (2) are clear.

(3) Let $J$ be a sink of $f$ and let $U$ be the largest open interval with $J \subseteq U \subseteq I$ such that $f^{n}(U) \subseteq I$ for all $n \geq 0$. The maximality of $U$ and lemma 3.1 ensure that $U$ is the maximal sink of $f$ containing $J$. If $J_{1}$ and $J_{2}$ are maximal sinks of $f$ with $J_{1} \neq J_{2}$ then by (1) we have $J_{1} \cap J_{2}=\varnothing$.

(4) Let $L$ be a homterval of $f$ and let $U$ be the largest open interval with $L \subseteq U \subseteq I$ such that $f^{n}(U) \subseteq I$ for all $n \geq 0$. $U$ cannot be contained in a sink of $f$ since $L$ is a homterval of $f$. Hence $U$ is the maximal homterval of $f$ containing $L$. If $L_{1}$ and $L_{2}$ are maximal homtervals of $f$ with $L_{1} \neq L_{2}$ then by (1) we have $L_{1} \cap L_{2}=\varnothing$.

Put

$$
\text { Sink }(f)=\left\{x \in I: f^{m}(x) \in J \text { for some sink } J \text { of } f \text { and some } m \geq 0\right\}
$$

and

Homt $(f)=\left\{x \in I: f^{m}(x) \in L\right.$ for some homterval $L$ of $f$ and some $\left.m \geq 0\right\}$;

then Sink $(f)$ and Homt $(f)$ are both open and by proposition 3.2 (2) we have $f(\operatorname{Sink}(f)) \subseteq \operatorname{Sink}(f)$ and $f($ Homt $(f)) \subseteq$ Homt $(f)$. Moreover, it is easy to see that Sink $(f)$ and Homt $(f)$ are both $f$-biinvariant.

Proposition 3.3 (cf. [16, proposition 4.3]). (1) Sink $(f) \cap \operatorname{Homt}(f)=\varnothing$.

(2) If $L \subseteq$ Homt $(f)$ is a non-empty open interval then $L$ is a homterval of $f$.

(3) If $L$ is a homterval of $f$ then $f^{n}(L) \cap f^{k}(L)=\varnothing$ whenever $0 \leq k<n$.

(4) If $x \in \operatorname{Sink}(f)$ then there exists $q \geq 1$ such that $\lim _{n \rightarrow \infty} f^{n q}(x)$ exists.

Proof. (1) Suppose that $\operatorname{Sink}(f) \cap \operatorname{Homt}(f) \neq \varnothing$. Then there exist a sink $J$ of $f$ and a homterval $L$ of $f$ such that $L \cap J \neq \varnothing$. Lemma 3.1 gives us then that $J \cup L$, and thus also $L$, is contained in a sink of $f$. But this is not possible since $L$ is a homterval of $f$. 
(2) Let $L \subseteq$ Homt $(f)$ be a non-empty open interval. Then for each $n \geq 0$ we have $f^{n}(L) \subseteq I$ and by $(1) f^{n}(L)$ is not contained in any sink of $f$. Hence $L$ is a homterval of $f$.

(3) Let $0 \leq k \leq n$; applying lemma 3.1 with $U=f^{k}(L)$ and $m=n-k$ shows that if $L$ is a homterval of $f$ then $f^{k}(L) \cap f^{n}(L)=\varnothing$.

(4) Suppose that $x \in \operatorname{Sink}(f)$; then by proposition 3.2(2) there exist $k \geq 0, m \geq 1$ and a sink $J$ of $f$ such that $f^{k m}(x) \in J$ and $f^{m}(J) \subseteq J$. Since $f^{m}$ is continuous and monotone on $J$ this gives us that for each $n \geq k, f^{n m}(x) \in J$ and that the sequence $\left\{f^{2 n m}(x)\right\}_{n \geq k}$ is monotone. Hence $\lim _{n \rightarrow \infty} f^{2 n m}(x)$ exists.

Put $M(f)=\left\{x \in I: f^{m}(x)=\omega\right.$ for some $\left.m \geq 0\right\}$. Note that $M(f)$ and thus also $\overline{M(f)}$ are $f$-almost-invariant and that $I-\overline{M(f)}$ is the largest open set $U \subseteq I$ such that $f^{n}(U) \cap S(f)=\varnothing$ for all $n \geq 0$.

Proposition 3.4 (cf. [16, proposition 4.2]). $I-\overline{M(f)}=\operatorname{Sink}(f) \cup$ Homt $(f)$.

Proof. Suppose that $x \in \operatorname{Sink}(f) \cup \operatorname{Homt}(f)$; then there exists $m \geq 0$ such that $f^{m}(x) \in J$ where $J$ is either a sink or a homterval of $f$. Thus $f^{m}((x-\varepsilon, x+\varepsilon)) \subseteq J$ for some $\varepsilon>0$ and hence $f^{n}((x-\varepsilon, x+\varepsilon)) \subseteq I$ for each $n \geq 0$. This shows that $x \in I-\overline{M(f)}$. On the other hand, let $U \subseteq I-\overline{M(f)}$ be a non-empty open interval. Then $f^{n}(U) \subseteq I$ for all $n \geq 0$. Thus either $U$ is a homterval of $f$ or there exists $m \geq 0$ such that $f^{m}(U)$ is contained in some sink of $f$. Hence $U \subseteq \operatorname{Sink}(f) \cup$ Homt $(f)$.

The following lemma will be useful later.

LEMMA 3.5. If $U \subseteq \overline{M(f)}$ is non-empty, open and $f$-almost-invariant then $U \cap S(f) \neq \varnothing$.

Proof. Suppose that $U \subseteq \overline{M(f)}$ is non-empty, open and $f$-almost-invariant. Since $U \cap M(f) \neq \varnothing$ we have $\varphi \in f^{n}(U)=f^{n}\left(U-S\left(f^{n}\right)\right) \subseteq U$ for some $\varphi \in S(f)$ and $n \geq 0$.

\section{Topologically transitive cyclés and register-shifts}

This section is concerned with the definitions and some elementary properties of topologically transitive $f$-cycles and $f$-register-shifts.

We call $C \subseteq I$ an $f$-cycle if $C$ is $f$-almost-invariant and is the disjoint union of non-trivial closed intervals $B_{1}, \ldots, B_{m}(m \geq 1)$ such that whenever $1 \leq j \leq m$ and $U \subseteq C$ is non-empty and open then $f^{n}(U) \cap B_{j} \neq \varnothing$ for some $n \geq 1 . B_{1}, \ldots, B_{m}$ are then called the components of $C$.

Clearly, $I$ is an $f$-cycle. Note that $C$ being an $f$-cycle does not necessarily mean that $f$ cyclically permutes or even permutes the components of $C$. But if $f_{\mid C}$ is really 'continuous' in that there exists a continuous function $g: C \rightarrow C$ with $g(x)=f(x)$ for all $x \in I-S(f)$, then we can label the components $B_{1}, \ldots, B_{m}$ of the $f$-cycle $C$ in such a way that $f\left(B_{i}-S(f)\right) \subseteq B_{i+1}$ for $i=1, \ldots, m-1$ and $f\left(B_{m}-S(f)\right) \subseteq B_{1}$. In general however, the situation is much more complicated since, for a component $B$ of $C, f(B-S(f))$ is not necessarily contained in a single component of $C$.

For an $f$-cycle $C$ let $A(C, f)$ denote the set of points in $I$ which eventually 'end up' in the interior of $C$, i.e.

$$
A(C, f)=\left\{x \in I: f^{m}(x) \in \operatorname{int}(C) \text { for some } m \geq 0\right\} .
$$

Clearly, $A(C, f)$ is open and $f$-biinvariant. 
Lemma 4.1. Let $C$ and $K$ be f-cycles. Then:

(1) If int $(B \cap K)=\varnothing$ for some component $B$ of $C$ then int $(C \cap K)=\varnothing$.

(2) $A(C, f) \cap A(K, f)=\varnothing$ if and only if int $(C \cap K)=\varnothing$.

Proof. (1) Let $B$ be a component of $C$. If int $(C \cap K) \neq \varnothing$ then by definition we have $\varnothing \neq f^{n}$ (int $\left.(C \cap K)\right) \cap B=f^{n}$ (int $\left.(C \cap K)-S\left(f^{n}\right)\right) \cap B$ for some $n \geq 1$. But since for each $m \geq 1, f^{m}$ (int $\left.(C \cap K)-S\left(f^{m}\right)\right)$ is an open subset of $K$ it follows that int $(B \cap K) \neq \varnothing$.

(2) Let $x \in A(C, f) \cap A(K, f)$; then $f^{n}(x) \in$ int $(C)$ and $f^{m}(x) \in$ int (K) for some $n, m \geq 0$. Putting $k=\max \{n, m\}$ we have $f^{k}(x) \in$ int $(C \cap K)$. The proof of the converse is trivial since $\varnothing \neq \operatorname{int}\left(K^{\prime}\right) \subseteq A\left(K^{\prime}, f\right)$ for each $f$-cycle $K^{\prime}$.

In order to analyse the structure of $\overline{M(f)}$ we will be interested in $f$-cycles contained in $\overline{M(f)}$. Looking for $f$-cycles which are 'minimal' leads us to the following definition. We say that an $f$-cycle $C$ is topologically transitive if whenever $F$ is a closed, $f$-almost-invariant subset of $C$ then either $F=C$ or $\operatorname{int}(F)=\varnothing$.

For $x \in I$ put $O_{f}(x)=\left\{f^{n}(x): n \geq 0\right\} ; O_{f}(x)$ is called the orbit of $x$ (under $f$ ).

Proposition 4.2 (cf. [18, theorem 5.8]. Let $C \subseteq I$ be an f-cycle. Then the following are equivalent:

(1) $C$ is a topologically transitive $f$-cycle.

(2) If $U \subseteq C$ is non-empty, open and f-almost-invariant then $\bar{U}=C$.

(3) If $U, V \subseteq C$ are non-empty and open then $f^{n}(U) \cap V \neq \varnothing$ for some $n \geq 0$.

(4) If $U, V \subseteq C$ are non-empty and open then $f^{-n}(U) \cap V \neq \varnothing$ for some $n \geq 0$.

(5) $\left\{x \in C: \overline{O_{f}(x)} \neq C\right\}$ is of the first category.

(6) $\overline{O_{f}(x)}=C$ for some $x \in C$.

Proof. (1) $\Rightarrow(2)$ Suppose that $U \subseteq C$ is non-empty, open and $f$-almost-invariant. Then $\bar{U} \subseteq C$ is closed and $f$-almost-invariant; hence since $C$ is topologically transitive we have $\bar{U}=C$.

(2) $\Rightarrow(3)$ Suppose that $U, V \subseteq C$ are non-empty and open. Put $A=$ $\bigcup_{n \geq 0} f^{n}\left(U-S\left(f^{n}\right)\right)$. Then $A$ is a non-empty, open and $f$-almost-invariant subset of $C$, and thus by (2) $\bar{A}=C$. Hence $f^{n}(U) \cap V \neq \varnothing$ for some $n \geq 0$.

$(3) \Rightarrow(4)$ This is clear.

(4) $\Rightarrow$ (5) Let $U_{1}, U_{2}, \ldots$ be a countable base for the relative topology on $C$ and let $x \in C$. Then $\overline{O_{f}(x)} \neq C$ if and only if $O_{f}(x) \cap U_{n}=\varnothing$ for some $n \geq 1$. Thus

$$
\begin{aligned}
\left\{x \in C: \overline{O_{f}(x)} \neq C\right\} & =C \cap \bigcup_{n \geq 1} \bigcap_{m \geq 0} f^{-m}\left(I^{\prime}-U_{n}\right) \\
& \subseteq \bigcup_{n \geq 1}\left(I-\left(\bigcup_{m \geq 0} f^{-m}\left(U_{n}\right) \cap \operatorname{int}(C)\right)\right) \cap C .
\end{aligned}
$$

Since $\bigcup_{m \geq 0} f^{-m}\left(U_{n}\right) \cap \operatorname{int}(C)$ is open and by (4) dense in $C$ the set of points $\left\{x \in C: \overline{O_{f}(x)} \neq C\right\}$ is of the first category.

$(5) \Rightarrow(6)$ This is clear since $C$ is a set of the second category.

(6) $\Rightarrow(1)$ Let $x \in C$ with $\overline{O_{f}(x)}=C$ and let $F \subseteq C$ be closed and $f$-almost-invariant with int $(F) \neq \varnothing$. Then we have $f^{k}(x) \in F$ for some $k \geq 0$, and thus $\left\{f^{n}(x): n \geq k\right\} \subseteq F$. Since $C$ has no isolated points it follows that $C=\overline{O_{f}(x)}=\overline{\left\{f^{n}(x): n \geq k\right\}}=F$. 
Proposition 4.3 (cf. [16, proposition 2.1]). Let $C$ and $K$ be topologically transitive f-cycles. Then:

(1) $A(C, f) \cap(\operatorname{Sink}(f) \cup$ Homt $(f))=\varnothing$.

(2) $C \subseteq \overline{M(f)}$.

(3) int $(C) \cap S(f) \cap(a, b) \neq \varnothing$.

(4) Either $C=K$ or $A(C, f) \cap A(K, f)=\varnothing$.

Proof. (1) Suppose that $A(C, f) \cap \operatorname{Homt}(f) \neq \varnothing$. Then there exists a homterval $L$ of $f$ with $L \subseteq C$. By proposition 3.3(3) we would then have $L \cap f^{n}(f(L))=\varnothing$ for all $n \geq 0$ which by proposition 4.2 is not possible.

Suppose next that $A(C, f) \cap \operatorname{Sink}(f) \neq \varnothing$. Then int $(C) \cap \operatorname{Sink}(f) \neq \varnothing$ and by proposition 3.3(4) we would have

$$
\text { int }(C) \cap \operatorname{Sink}(f) \subseteq\left\{x \in C: \overline{O_{f}(x)} \neq C\right\} .
$$

Again this contradicts proposition 4.2 .

(2) By (1) and proposition 3.4 we have

Hence $C \subseteq \overline{M(f)}$.

$$
A(C, f) \subseteq I-(\operatorname{Sink}(f) \cup \operatorname{Homt}(f))=\overline{M(f)} .
$$

(3) Since int $(C) \cap(a, b)$ is an $f$-almost-invariant subset of $\overline{M(f)}$, lemma 3.5 gives us that int $(C) \cap S(f) \cap(a, b) \neq \varnothing$.

(4) Suppose that $A(C, f) \cap A(K, f) \neq \varnothing$. Then by lemma 4.1(2) we have int $(C \cap K) \neq \varnothing$. Since $C \cap K$ is closed and $f$-almost-invariant it follows from the topological transitivity of $C$ and $K$ that $C=C \cap K=K$.

Examples of piecewise monotone functions having topologically transitive cycles are easily found. For instance, let $g \in \mathcal{N}(I)$ be given by $g(x)=2 x-a$ if $a<x<$ $(a+b) / 2$ and $g(x)=2 x-b$ if $(a+b) / 2<x<b$. As it is shown in corollary $7.2, I$ is then a topologically transitive $g$-cycle. In general we cannot describe the asymptotic behaviour of elements of $\mathcal{N}(I)$ in terms of sinks, homtervals and topologically cycles alone. For $\mu \in(0,4)$ let $f_{\mu} \in \mathcal{N}([0,1])$ be given by $f_{\mu}(x)=\mu x(1-x)$ for all $x \in$ $(0,1)-\left\{\frac{1}{2}\right\}$. Then for a certain value of $\mu(\mu \approx 3.56994)$ we have $\operatorname{Sink}\left(f_{\mu}\right)=$ Homt $\left(f_{\mu}\right)=\varnothing$ and there is no topologically transitive $f_{\mu}$-cycle. (See for instance [6] and $[3$, theorem 2.6]). In [16, $\S 7]$ a function $g \in \mathcal{N}(I)$ is explicitly constructed having neither sinks, homtervals nor topologically transitive $g$-cycles. In both cases there exists a decreasing sequence of cycles $\left\{K_{n}\right\}_{n \geq 1}$ such that int $\left(\bigcap_{n \geq 1} K_{n}\right)=\varnothing$. This suggests the following definition.

We call $R \subseteq I$ an $f$-register-shift if int $(R)=\varnothing$ and if there exists a decreasing sequence $\left\{K_{n}\right\}_{n \geq 1}$ of $f$-cycles $K_{n}$ contained in $\overline{M(f)}$ such that $R=\bigcap_{n \geq 1} K_{n}$ $\left(\left\{K_{n}\right\}_{n \geq 1}\right.$ is said to be decreasing if $K_{n+1} \subseteq K_{n}$ for each $\left.n \geq 1\right)$; we then say that $\left\{K_{n}\right\}_{n \geq 1}$ is a generator for the $f$-register-shift $R$.

Proposition 4.4. Let $\left\{K_{n}\right\}_{n \geq 1}$ be a generator for some f-register-shift $R$ and let $\left\{K_{n}^{\prime}\right\}_{n \geq 1}$ be a generator for some f-register-shift $R^{\prime}$. Then:

(1) If $C$ is a topologically transitive f-cycle then $A(C, f) \cap A\left(K_{m}, f\right)=\varnothing$ for some $m \geq 1$.

(2) $S(f) \cap(a, b) \cap \bigcap_{n \geq 1}$ int $\left(K_{n}\right) \neq \varnothing$.

(3) $R \neq R^{\prime}$ if and only if $A\left(K_{m}, f\right) \cap A\left(K_{m}^{\prime}, f\right)=\varnothing$ for some $m \geq 1$. 
Proof. (1) Suppose that $C$ is a topologically transitive $f$-cycle and assume that $A(C, f) \cap A\left(K_{n}, f\right) \neq \varnothing$ for all $n \geq 1$. Then by lemma 4.1(2) we would have int $\left(C \cap K_{n}\right) \neq \varnothing$ and thus $C \subseteq K_{n}$ for all $n \geq 1$ (because $C$ is topologically transitive). But this is not possible since int $\left(\bigcap_{n \geq 1} K_{n}\right)=\varnothing$.

(2) Since int $\left(K_{n}\right) \cap(a, b)$ is an $f$-almost-invariant subset of $\overline{\boldsymbol{M}(f)}$ we have by lemma 3.5, int $\left(K_{n}\right) \cap S(f) \cap(a, b) \neq \varnothing$ for each $n \geq 1$. Hence

(because $S(f)$ is finite).

$$
S(f) \cap(a, b) \cap \bigcap_{n \geq 1} \operatorname{int}\left(K_{n}\right) \neq \varnothing
$$

(3) Let $x \in R-R^{\prime}$; then $x \in R-K_{j}^{\prime}$ for some $j \geq 1$. For $n \geq 1$ let $B_{n}$ be the unique component of $K_{n}$ with $x \in B_{n}$. Since int $(R)=\varnothing$ we have $\{x\}=\bigcap_{n \geqslant 1} B_{n}$; hence $B_{m} \cap K_{j}^{\prime}=\varnothing$ for some $m \geq j$. Now lemma 4.1 gives that

$$
\text { int }\left(K_{m} \cap K_{m}^{\prime}\right) \subseteq \text { int }\left(K_{m} \cap K_{j}^{\prime}\right)=\varnothing \text {, }
$$

and thus $A\left(K_{m}, f\right) \cap A\left(K_{m}^{\prime}, f\right)=\varnothing$. Conversely, if $A\left(K_{m}, f\right) \cap A\left(K_{m}^{\prime}, f\right)=\varnothing$ for some $m \geq 1$ then int $\left(K_{m}\right) \cap$ int $\left(K_{m}^{\prime}\right)=\varnothing$, and thus by (2) $R \neq R^{\prime}$.

Let $R$ be an $f$-register-shift; put $A(R, f)=\bigcap_{C \in \mathscr{C}(R)} A(C, f)$ where $\mathscr{C}(R)$ is the set of $f$-cycles $C$ with $R \subseteq C$. Let $\left\{K_{n}\right\}_{n \geq 1}$ be a generator for $R$. One can show that in general $A(R, f)=\bigcap_{n \geq 1} A\left(K_{n}, f\right)$ is not true. However, we will prove in $\S 5$ (theorem 5.9(2)) that there exists a generator $\left\{K_{n}\right\}_{n \geq 1}$ for $R$ such that $A(R, f)=\bigcap_{n \geq 1} A\left(K_{n}, f\right)$. In particular, this shows that $A(R, f)$ is a $G_{\delta^{-}}$-set (i.e. $A(R, f)$ can be written as a countable intersection of open sets).

In $\S 6$ we will study some more properties of $f$-register-shifts. We will show that each $f$-register-shift $R$ is a Cantor-like set, that $R-Q \subseteq f(R-S(f)) \subseteq R$ where $Q$ is a finite set of points in $I$, that the orbit of each point in $R-M(f)$ is dense in $R$ and that each element of $A(R, f)$ is attracted to $R$.

\section{The main result}

We now come to the main result.

THEOREM 5.1. Let $C_{1}, \ldots, C_{r}$ be the topologically transitive f-cycles, let $R_{1}, \ldots, R_{s}$ be the f-register-shifts and for $1 \leq i \leq s$ let $\left\{K_{n}^{(i)}\right\}_{n \geq 1}$ be a generator for $R_{i}$. Then

$$
r+s \leq \operatorname{card}(S(f) \cap(a, b)),
$$

and there exists $m \geq 1$ such that for each $n \geq m$ the open and f-biinvariant sets $A\left(C_{1}, f\right), \ldots, A\left(C_{r}, f\right), A\left(K_{n}^{(1)}, f\right), \ldots, A\left(K_{n}^{(s)}, f\right)$, Sink $(f)$, Homt $(f)$ are disjoint and their union is dense in $I$.

Proof. The proof of theorem 5.1, which is based on a couple of lemmas and propositions, can be found at the end of this section.

Let $C_{1}, \ldots, C_{r}$ be the topologically transitive $f$-cycles and let $R_{1}, \ldots, R_{s}$ be the $f$-register-shifts. Put

$$
G(f)=A\left(C_{1}, f\right) \cup \ldots \cup A\left(C_{r}, f\right) \cup A\left(R_{1}, f\right) \cup \ldots \cup A\left(R_{s}, f\right) \cup \operatorname{Sink}(f) \cup \operatorname{Homt}(f) .
$$

Theorem 5.9(2) will show that for each $1 \leq i \leq s$ there exists a generator $\left\{K_{n}^{(i)}\right\}_{n \geq 1}$ for $R_{i}$ such that $A\left(R_{i}, f\right)=\bigcap_{n \geq 1} A\left(K_{n}^{(i)}, f\right)$. Hence by theorem 5.1 and the Baire category theorem the $G_{\delta}$-set $G(f)$ is dense in $I$. 
The following example shows that $I-G(f)$ (which is $f$-biinvariant) can have a quite complicated structure. Consider Newton's Method for determining the zeros of a polynomial $p$. By identifying $\overline{\mathbb{P}}$ with $I$ we obtain a discrete dynamical system on $I$ represented by some $g \in \mathcal{N}(I)$. If $p$ is an $n$-th degree polynomial with $n \geq 4$ having real roots, then it follows from a result by Barna [1] that Sink $(g)$ is dense in $I$ (and thus there are neither homtervals of $g$, topologically transitive $g$-cycles nor $g$-register-shifts) and that $I-G(g)$ contains a Cantor-like set. Furthermore, it should be noted that the action of $f$ restricted to $I-G(f)$ can be very complex. In the continuous case (i.e. if there exists a continuous function $g$ on $I$ into itself with $g(x)=f(x)$ for all $x \in I-S(f))$ the asymptotic behaviour of $f$ on $I-G(f)$ is analysed in [16] by using certain factors of $f$ which essentially 'kill off' $G(f)$.

For $\varphi \in S(f)$ and $n \geq 1$ put

$$
I_{n}(\varphi)=\left(\varphi-\frac{1}{n}, \varphi+\frac{1}{n}\right) \cap I
$$

and

$$
L_{n}(\varphi)=\bigcup_{m \geqslant 0} f^{m}\left(I_{n}(\varphi)-S\left(f^{m}\right)\right) .
$$

Then $L_{n}(\varphi)$ is non-empty, open and $f$-almost-invariant and we have $L_{n+1}(\varphi) \subseteq L_{n}(\varphi)$ for all $n \geq 1$. Furthermore, for $\varphi \in S(f)$ put

$$
\Delta(\varphi)=\left\{x \in I: f^{m}(x)=\varphi \text { for some } m \geq 0\right\} ;
$$

note that $\Delta(\varphi)$ and thus also $\overline{\Delta(\varphi)}$ and int $\overline{(\Delta(\varphi))}$ are $f$-almost-invariant.

The proof of theorem 5.1 will consist in showing that for each $\varphi \in S(f)$ with int $\overline{(\Delta(\varphi))} \neq \varnothing$ there exists $m \geq 1$ such that either $\overline{L_{m}(\varphi)}$ is a topologically transitive $f$-cycle or $\left\{\overline{L_{n}(\varphi)}\right\}_{n \geq m}$ is a generator for some $f$-register-shift $R$. In order to prove that (for sufficiently large $n$ ) $\overline{L_{n}(\varphi)}$ is an $f$-cycle we will first study some properties of certain (connected) components of open, $f$-almost-invariant subsets of $I$.

Let $U \subseteq I$ be open; a component $J$ of $U$ is called regular if there exists a component $L$ of $U$ with $L \cap S(f) \neq \varnothing$ such that $f^{k}(L) \cap J \neq \varnothing$ for some $k \geq 0$. Let $\varphi \in S(f)$ and $n \geq 1$; note that each component of $L_{n}(\varphi)$ is regular.

LEMMA 5.2. Let $U \subseteq I$ be non-empty, open and f-almost-invariant and let $J$ be a regular component of $U$. Then there exists a component $K$ of $U-S(f)$ and $m \geq 0$ such that $f^{m}(K) \subseteq J, K \cap S\left(f^{m}\right)=\varnothing$ and $K \subseteq L$ for some component $L$ of $U$ with $L \cap S(f) \neq \varnothing$.

Proof. Let

$$
m=\min \left\{k \geq 0: f^{k}(L) \cap J \neq \varnothing \text { for some component } L \text { of } U \text { with } L \cap S(f) \neq \varnothing\right\} .
$$

Choose a component $L$ of $U$ with $L \cap S(f) \neq \varnothing$ such that $f^{m}(L) \cap J \neq \varnothing$. Since $U$ is $f$-almost-invariant there exists a component $K$ of $L-S(f)$ such that $f^{m}(K) \subseteq J$. Then $K$ is a component of $U-S(f)$ and by the choice of $m$ we have $K \cap S\left(f^{m}\right)=\varnothing$.

LEMMA 5.3. Let $U \subseteq \overline{M(f)}$ be non-empty, open and f-almost-invariant. Then the set of the regular components of $U$ is non-empty and finite.

Proof. By Lemma 3.5 we have $U \cap S(f) \neq \varnothing$; hence we can find at least one regular component of $U$. Let $Y$ be the set of the components $K$ of $U-S(f)$ which are 
contained in some component $L$ of $U$ with $L \cap S(f) \neq \varnothing . \mathscr{Y}$ is finite because we have for all $K \in \mathscr{Y}$ and $\varphi \in S(f), \bar{K} \cap S(f) \neq \varnothing$ and card $(\{K \in \mathscr{Y}: \varphi \in \bar{K}\}) \leq 2$. Since $U \subseteq \overline{M(f)}$ we can find $N \geq 1$ such that for all $K \in \mathscr{Y}$ there exists $0 \leq n \leq N$ with $f^{n}(K) \cap S(f) \neq \varnothing$. Now let $J$ be a regular component of $U$. By lemma 5.2 there exist $K \in Y$ and $m \geq 0$ such that $f^{m}(K) \subseteq J, K \cap S\left(f^{m}\right)=\varnothing$ and $m<N$. Since $J$ is the unique component of $U$ with $f^{m}(K) \subseteq J$, this shows that there are at most $N \cdot$ card $(\mathscr{Y})$ regular components of $U$.

LEMMA 5.4. Let $U, U^{\prime}$ be open and f-almost-invariant subsets of $I$ with $U^{\prime} \subseteq U$ and $U \cap S(f)=U^{\prime} \cap S(f)$. Then each regular component of $U$ contains at least one regular component of $U^{\prime}$.

Proof. Let $J$ be a regular component of $U$. By lemma 5.2 there exist a component $K$ of $U-S(f)$ and $m \geq 0$ such that $f^{m}(K) \subseteq J, K \cap S\left(f^{m}\right)=\varnothing$ and $K \subseteq L$ for some component $L$ of $U$ with $L \cap S(f) \neq \varnothing$. Since $U \cap S(f)=U^{\prime} \cap S(f)$ we can find a component $L^{\prime}$ of $U^{\prime}$ with $L^{\prime} \cap S(f) \neq \varnothing$ such that $L^{\prime} \subseteq L$ and $L^{\prime} \cap K \neq \varnothing$. Then since $U^{\prime}$ is $f$-almost-invariant $f^{m}\left(L^{\prime} \cap K\right)$ is a non-empty open interval which is contained in $U^{\prime} \cap J$. Let $J^{\prime}$ be the unique component of $U^{\prime}$ with $f^{m}\left(L^{\prime} \cap K\right) \subseteq J^{\prime}$. Then $J^{\prime} \subseteq J$ and $J^{\prime}$ is regular.

Next we want to show that if $\varphi \in S(f)$ with int $\overline{(\Delta(\varphi))} \neq \varnothing$ and $n$ is sufficiently large then $\overline{L_{n}(\varphi)}$ is an $f$-cycle. For this we need the following lemma.

LemMA 5.5. Let $\varphi \in S(f)$ with int $\overline{(\Delta(\varphi))} \neq \varnothing$. Then:

(1) If $U \subseteq \overline{\Delta(\varphi)}$ is non-empty, open and f-almost-invariant, then $L_{n}(\varphi) \subseteq U$ for some $n \geq 1$.

(2) There exists $m \geq 1$ such that $L_{n}(\varphi) \subseteq \overline{\Delta(\varphi)}$ and

$$
L_{n}(\varphi) \cap S(f)=L_{m}(\varphi) \cap S(f) \quad \text { for all } n \geq m .
$$

Proof. (1) Suppose that $U \subseteq \overline{\Delta(\varphi)}$ is non-empty, open and $f$-almost-invariant. Since $\Delta(\varphi) \cap U \neq \varnothing$ we have $\varphi \in f^{j}\left(U-S\left(f^{j}\right)\right) \subseteq U$ for some $j \geq 0$. Hence there exists $n \geq 1$ such that $I_{n}(\varphi) \subseteq U$. Again using that $U$ is $f$-almost-invariant gives us that $L_{n}(\varphi) \subseteq U$.

(2) Since int $\overline{(\Delta(\varphi))}$ is $f$-almost-invariant and $S(f)$ is finite there exists by (1) $m \geq 1$ such that $L_{n}(\varphi) \subseteq \overline{\Delta(\varphi)}$ and $L_{n}(\varphi) \cap S(f)=L_{m}(\varphi) \cap S(f)$ for all $n \geq m$.

Proposition 5.6. Let $\varphi \in S(f)$ with int $\overline{(\Delta(\varphi))} \neq \varnothing$. Then for all sufficiently large $n \geq 1$ the number of components of $L_{n}(\varphi)$ is finite and $\overline{L_{n}(\varphi)}$ is an f-cycle contained in $\overline{\Delta(\varphi)}$.

Proof. By lemma 5.5(2) there exists $m \geq 1$ such that $L_{n}(\varphi) \subseteq \overline{\Delta(\varphi)}$ and $L_{n}(\varphi) \cap S(f)=$ $L_{m}(\varphi) \cap S(f)$ for all $n \geq m$. Now let $n \geq m$. Since each component of $L_{n}(\varphi)$ is regular lemma 5.3 immediately gives us that the number of components of $L_{n}(\varphi)$ is finite. Hence $\overline{L_{n}(\varphi)}$ can be written as a disjoint union of non-trivial closed intervals $B_{1}, \ldots, B_{p}$ (with $p \geq 1$ ). Let $U \subseteq \overline{L_{n}(\varphi)}$ be open and non-empty. In order to prove that $\overline{L_{n}(\varphi)}$ is an $f$-cycle it remains to show that for each $1 \leq i \leq p$ we have $B_{i} \cap f^{k}(U) \neq$ $\varnothing$ for some $k \geq 1$. Let $1 \leq i \leq p$ and put $V=\bigcup_{k \geq 1} f^{k}\left(U-S\left(f^{k}\right)\right)$. Then $V$ is open and (since $V$ and $\bar{L}_{n}(\varphi)$ are both $f$-almost-invariant) we have $V \subseteq \overline{L_{n}(\varphi)} \subseteq \overline{\Delta(\varphi)}$. Hence by lemma $5.5(1)$ there exists $j \geq n$ with $L_{j}(\varphi) \subseteq V$. $B_{i}$ contains at least one 
component $J$ of $L_{n}(\varphi)$, which is regular. By lemma 5.4 there exists a component $J^{\prime}$ of $L_{j}(\varphi)$ with $J^{\prime} \subseteq J \subseteq B_{i}$. Hence $J^{\prime} \subseteq B_{i} \cap V$ and therefore $B_{i} \cap f^{k}(U) \neq \varnothing$ for some $k \geq 1$.

Proposition 5.7. Let $L_{\infty}=\bigcap_{n \geq 1} \overline{L_{n}(\varphi)}$ for some $\varphi \in S(f)$ with int $\overline{(\Delta(\varphi)} \neq \varnothing$. Then:

(1) If int $\left(L_{\infty}\right) \neq \varnothing$ then $L_{\infty}$ is a topologically transitive f-cycle and $L_{\infty}=\overline{L_{m}(\varphi)}$ for some $m \geq 1$.

(2) If int $\left(L_{\infty}\right)=\varnothing$ then $L_{\infty}$ is an $f$-register-shift and for all sufficiently large $m$, $\left\{\overline{L_{n}(\varphi)}\right\}_{n \geq m}$ is a generator for $L_{\infty}$ with $A\left(L_{\infty}, f\right)=\bigcap_{n \geq m} A\left(\overline{L_{n}(\varphi)}, f\right)$.

Proof. (1) Put $V=\operatorname{int}\left(L_{\infty}\right)$ and suppose that $V$ is non-empty. By lemma 5.5(2) we have $V \subseteq \overline{L_{k}(\varphi)} \subseteq \overline{\Delta(\varphi)}$ for some $k \geq 1$ and by lemma 5.5(1) we can find $m \geq 1$ with $L_{m}(\varphi) \subseteq V$. Thus $\overline{L_{n}(\varphi)}=\overline{L_{m}(\varphi)}=L_{\infty}$ for all $n \geq m$, and so by proposition $5.6 \overline{L_{m}(\varphi)}$ is an $f$-cycle contained in $\overline{\Delta(\varphi)}$. Now let $U \subseteq \overline{L_{m}(\varphi)}$ be non-empty, open and $f$-almost-invariant. Again by lemma $5.5(1)$ we have $L_{j}(\varphi) \subseteq U$ for some $j \geq m$ and hence $\overline{L_{j}(\varphi)}=\bar{U}=\overline{L_{m}(\varphi)}$. Therefore by proposition $4.2, \overline{L_{m}(\varphi)}$ is a topologically transitive $f$-cycle.

(2) Suppose that int $\left(L_{\infty}\right)=\varnothing$. By proposition 5.6 there exists $m \geq 1$ such that for each $n \geq m \overline{L_{n}(\varphi)}$ is an $f$-cycle contained in $\overline{\Delta(\varphi)} \subseteq \overline{M(f)}$. Hence $L_{\infty}$ is an $f$-register-shift and $\left\{\overline{L_{n}(\varphi)}\right\}_{n \geq m}$ is a generator for $L_{\infty}$. In order to show that $A\left(L_{\infty}, f\right)=\bigcap_{n \geq m} A\left(\overline{L_{n}(\varphi)}, f\right)$, consider an $f$-cycle $K$ with $L_{\infty} \subseteq K$; put $U=$ int $(K) \cap \operatorname{int}(\overline{\Delta(\varphi)})$. Then $U$ is non-empty because $\varphi \in L_{\infty} \cap \operatorname{int}(\overline{\Delta(\varphi)}) \subseteq K \cap$ int $(\overline{\Delta(\varphi)})$. Moreover, $U$ is open and $f$-almost-invariant; hence by lemma 5.5(1) we have $L_{n}(\varphi) \subseteq U \subseteq K$ and thus int $\left(\overline{L_{n}(\varphi)}\right) \subseteq$ int $(K)$ for some $n \geq m$. Therefore $A\left(\overline{L_{n}(\varphi)}, f\right) \subseteq A(K, f)$ which shows that $A\left(L_{\infty}, f\right)=\bigcap_{n \geq m} A\left(\overline{L_{n}(\varphi)}, f\right)$.

LEMMA 5.8. Let $U \subseteq \overline{M(f)}$ be non-empty, open and f-almost-invariant. Then there exists $\varphi \in S(f)$ with int $(\overline{\Delta(\varphi)}) \neq \varnothing$ such that $L_{n}(\varphi) \subseteq U$ for some $n \geq 1$.

Proof. $\bigcup_{\varphi \in S(f)}$ int $(\overline{\Delta(\varphi)})$ is dense in $\overline{M(f)}$ since

$$
\overline{M(f)}-\bigcup_{\varphi \in S(f)} \operatorname{int} \overline{(\Delta(\varphi))}=\bigcup_{\varphi \in S(f)} \overline{\Delta(\varphi)}-\bigcup_{\varphi \in S(f)} \operatorname{int}(\overline{\Delta(\varphi)}) \subseteq \bigcup_{\varphi \in S(f)} \partial(\overline{\Delta(\varphi)}) .
$$

Hence there exists $\varphi \in S(f)$ with int $(\overline{\Delta(\varphi)}) \cap U \neq \varnothing$, and thus by lemma 5.5(1) we have $L_{n}(\varphi) \subseteq U$ for some $n \geq 1$.

Proof of Theorem 5.1. By propositions 4.3 and 4.4 there exists $m \geq 1$ such that for all $n \geq m$ the open and $f$-biinvariant sets $A\left(C_{1}, f\right), \ldots, A\left(C_{r}, f\right)$, $A\left(K_{n}^{(1)}, f\right), \ldots, A\left(K_{n}^{(s)}, f\right)$, Sink $(f)$, Homt $(f)$ are disjoint and each of them apart from Sink $(f)$ and Homt $(f)$ contains at least one element of $S(f) \cap(a, b)$. Thus $r+s \leq \operatorname{card}(S(f) \cap(a, b))$. For $n \geq m$ put

$$
\begin{gathered}
G_{n}=I-\left(A\left(C_{1}, f\right) \cup \ldots \cup A\left(C_{r}, f\right) \cup A\left(K_{n}^{(1)}, f\right) \cup \ldots \cup A\left(K_{n}^{(s)}, f\right) \cup \operatorname{Sink} k,(f)\right. \\
\cup \operatorname{Homt}(f)) .
\end{gathered}
$$

Then $G_{n}$ and thus int $\left(G_{n}\right)$ are $f$-almost-invariant, and by proposition 3.4 we have $G_{n} \subseteq \overline{M(f)}$. Lemma 5.8 and proposition 5.7 immediately give us that int $\left(G_{n}\right)=\varnothing$. 
The next theorem shows how each topologically transitive $f$-cycle and how for a given $f$-register-shift $R$ a generator $\left\{K_{n}\right\}_{n \geqslant 1}$ for $R$ with $A(R, f)=\bigcap_{n \geqslant 1} A\left(K_{n}, f\right)$ can be constructed; in particular, this implies that $A(R, f)$ is a $G_{\delta}$-set.

THEOREM 5.9. Let $C$ be a topologically transitive $f$-cycle and let $R$ be an $f$-register-shift. Then:

(1) $C=\bigcap_{n \geq 1} \overline{L_{n}(\varphi)}=\overline{L_{m}(\varphi)}$ for some $\varphi \in S(f)$ with int $\overline{\Delta(\varphi)} \neq \varnothing$.

(2) $R=\bigcap_{n \geq 1} \overline{L_{n}(\varphi)}$ and $\left\{\overline{L_{n}(\varphi)}\right\}_{n \geq m}$ is a generator for $R$ with $A(R, f)=$ $\bigcap_{n \geq m} A\left(\overline{L_{n}(\varphi)}, f\right)$ for some $\varphi \in S(f)$ with int $(\overline{\Delta(\varphi)}) \neq \varnothing$ and all large enough $m$.

Proof. (1) By proposition 4.3(2) we have $C \subseteq \overline{M(f)}$. Hence by lemma 5.8 there exists $\varphi \in S(f)$ with int $\overline{(\Delta(\varphi))} \neq \varnothing$ such that $L_{m}(\varphi) \subseteq C$ for some $m \geq 1$. Since $C$ is topologically transitive we have $C=\overline{L_{m}(\varphi)}=\bigcap_{n \geq 1} \overline{L_{n}(\varphi)}$.

(2) Let $\left\{K_{n}\right\}_{n \geq 1}$ be a generator for $R$. Since $S(f)$ is finite there exists, by lemma 5.8, $\varphi \in S(f)$ with int $\overline{(\Delta(\varphi))} \neq \varnothing$ such that for each $n \geq 1$ we have $L_{j}(\varphi) \subseteq K_{n}$ for some $j \geq n$. Hence propositions $4.4(3)$ and 5.7(2) give us that $R=\bigcap_{n \geq 1} \overline{L_{n}(\varphi)}$ and that for sufficiently large $m,\left\{\overline{L_{n}(\varphi)}\right\}_{n \geq m}$ is a generator for $R$ with $A(R, f)=$ $\bigcap_{n \geq m} A\left(\overline{L_{n}(\varphi)}, f\right)$.

6. More on register-shifts and topologically transitive cycles

In this section we will first study some more properties of $f$-register-shifts. In particular, we will show that each $f$-register-shift $R$ is a Cantor-like set, that $R-Q \subseteq f(R-S(f)) \subseteq R$ where $Q$ is a finite set of points in I and that the orbit of each point in $R-M(f)$ is dense in $R$. In the second part of this section we will prove that each topologically transitive $f$-cycle $C$ is in fact strongly transitive, i.e. for each open and non-empty subset $U$ of $C, \bigcup_{n=0}^{m} f^{n}\left(U-S\left(f^{n}\right)\right)$ is dense in $C$ for some $m \geq 0$.

For the sequel it will be convenient to consider a new dynamical system associated with $f$. For $x \in I$ and $\varepsilon>0$ let $B_{\varepsilon}(x, 1)=(x, x+\varepsilon) \cap I$ and $B_{\varepsilon}(x,-1)=(x-\varepsilon, x) \cap I$. For $A \subseteq I$ put

$$
A^{*}=\left\{(x, \alpha) \in I \times\{1,-1\}: B_{\varepsilon}(x, \alpha) \cap A \neq \varnothing \text { for each } \varepsilon>0\right\} .
$$

Let $K$ be an $f$-cycle; note that $(x, \alpha) \in K^{*}$ if and only if $B_{\varepsilon}(x, \alpha) \subseteq K$ for some $\varepsilon>0$. Let $(x, \alpha) \in I^{*}$ and $n \geq 0$; put $f^{n}(x, \alpha)=\lim _{y \downarrow x} f^{n}(y)$ if $\alpha=1$ and $f^{n}(x, \alpha)=$ $\lim _{y \uparrow x} f^{n}(y)$ if $\alpha=-1$. This is well defined and clearly we have $f^{n}(x, \alpha) \in I$. Moreover, there exists $\varepsilon>0$ such that $f^{n}$ is continuous and monotone on $B_{\varepsilon}(x, \alpha)$; put $\Pi^{n}(x, \alpha)=\alpha$ if $f^{n}$ is increasing on $B_{\varepsilon}(x, \alpha)$ and $\Pi^{n}(x, \alpha)=-\alpha$ if $f^{n}$ is decreasing on $B_{f}(x, \alpha)$. In the following we will write $f(x, \alpha)(\operatorname{resp} . \Pi(x, \alpha))$ instead of $f^{1}(x, \alpha)$ (resp. $\left.\Pi^{1}(x, \alpha)\right)$. Clearly $(f(x, \alpha), \Pi(x, \alpha)) \in I^{*}$ for each $(x, \alpha) \in I^{*}$.

Finally, let $F: I^{*} \rightarrow I^{*}$ be the mapping given by $F(x, \alpha)=(f(x, \alpha), \Pi(x, \alpha))$ and define the mapping $F^{n}: I^{*} \rightarrow I^{*}$ inductively by $F^{0}(x, \alpha)=(x, \alpha)$ and $F^{n}(x, \alpha)=$ $F\left(F^{n-1}(x, \alpha)\right)$ for all $n \geq 1$. It is not difficult to see that for all $(x, \alpha) \in I^{*}$ and $n, m \geq 0$ we have:

$$
\begin{aligned}
f^{m+n}(x, \alpha) & =f^{m}\left(f^{n}(x, \alpha), \Pi^{n}(x, \alpha)\right), \\
\Pi^{m+n}(x, \alpha) & =\Pi^{m}\left(f^{n}(x, \alpha), \Pi^{n}(x, \alpha)\right)
\end{aligned}
$$


and hence

$$
F^{n}(x, \alpha)=\left(f^{n}(x, \alpha), \Pi^{n}(x, \alpha)\right) .
$$

Furthermore, it is easily checked that for each $f$-cycle $K$ we have $F\left(K^{*}\right) \subseteq K^{*}$.

In order to show that each $f$-register-shift is a Cantor-like set we need the next two lemmas which will also be useful later. For $(x, \alpha) \in I^{*}$ put

for $B \subseteq I^{*}$ let

$$
O_{f}(x, \alpha)=\left\{f^{n}(x, \alpha): n \geq 0\right\},
$$

$$
O_{f}(B)=\left\{f^{n}(x, \alpha):(x, \alpha) \in B \text { and } n \geq 0\right\}
$$

and for an $f$-cycle $K$ put

$$
S(f, K)=\left\{(x, \alpha) \in K^{*}: x \in S(f)\right\} .
$$

Note that if $(x, \alpha) \in K^{*}$ for some $f$-cycle $K$ then $O_{f}(x, \alpha) \subseteq K$.

LEMMA 6.1. Let $K$ be an $f$-cycle and let $c, d \in K$ with $c<d$ and $(c, d) \subseteq K$. Then for each $n \geq 0$,

$$
\partial\left(f^{n}((c, d))\right) \subseteq O_{f}(S(f, K)) \cup\left\{f^{n}(c, 1), f^{n}(d,-1)\right\} .
$$

Proof. Let $n \geq 0$. If $u \in(c, d) \cap S\left(f^{n}\right)$ then there exists $0 \leq j<n$ with $f^{j}(u) \in S(f) \cap$ int $(K)$ and hence $f^{n}(u, \alpha) \in O_{f}(S(f, K))$ for all $\alpha \in\{1,-1\}$. Now let $(c, d) \cap S\left(f^{n}\right)=$ $\left\{u_{1}, \ldots, u_{m}\right\}$ with $u_{1}<u_{2}<\cdots<u_{m}$. Put $u_{0}=c$ and $u_{m+1}=d$. Then $f^{n}$ is continuous and monotone on each of the intervals $\left(u_{i}, u_{i+1}\right), i=0,1, \ldots, m$. Thus

$$
\begin{aligned}
\partial\left(f^{n}((c, d))\right) & =\partial\left(f^{n}\left((c, d)-S\left(f^{n}\right)\right)\right) \\
& \subseteq \bigcup_{1 \leq i \leq m} \partial\left(f^{n}\left(\left(u_{i}, u_{i+1}\right)\right)\right) \\
& \subseteq O_{f}(S(f, K)) \cup\left\{f^{n}(c, 1), f^{n}(d,-1)\right\} .
\end{aligned}
$$

LEMMA 6.2. Let $\left\{K_{n}\right\}_{n \geq 1}$ be a generator for some f-register-shift $R$ and let $(x, \alpha) \in I^{*}$ with $(x, \alpha) \in\left(K_{n}\right)^{*}$ for each $n \geq 1$. Then card $\left(O_{f}(x, \alpha)\right)=+\infty$.

Proof. Assume that card $\left(O_{f}(x, \alpha)\right)<+\infty$. Then also card $\left(\left\{F^{n}(x, \alpha): n \geq 0\right\}\right)<+\infty$, and hence there exist $q \geq 0$ and $p \geq 1$ such that $F^{p}(y, \beta)=(y, \beta)$ where $(y, \beta)=$ $F^{q}(x, \alpha)$. We have $(x, \alpha) \in\left(K_{n}\right)^{*}$ and thus $(y, \beta) \in\left(K_{n}\right)^{*}$ for each $n \geq 1$. Since by proposition 3.4, $K_{1} \cap \operatorname{Sink}(f)=\varnothing$ there exists $\varepsilon>0$ such that $f^{p}$ is continuous and increasing on $B_{2 \varepsilon}(y, \beta)$ and

$$
\beta \cdot f^{p}(z)>\beta \cdot z \quad \text { for all } z \in B_{2 \varepsilon}(y, \beta) .
$$

Hence for all $\delta>0$ there exists $m \geq 0$ such that $B_{\varepsilon}(y, \beta) \subseteq f^{m}\left(B_{\delta}(y, \beta)-S\left(f^{m}\right)\right)$; thus $B_{\varepsilon}(y, \beta) \subseteq K_{n}$ for each $n \geq 1$ which is not possible. Therefore, card $\left(O_{f}(x, \alpha)\right)=$ $+\infty$.

Let $\mathscr{C}=\left\{C_{n}\right\}_{n \geq 1}$ be a decreasing sequence of $f$-cycles and $m \geq 1$; we call a component $B$ of $C_{m} \mathscr{b}$-splitting if for some $k>m B$ contains at least two (distinct) components of $C_{k} . \mathscr{C}$ is said to be splitting if for all $n \geq 1$ each component of $C_{n}$ is $\mathscr{C}$-splitting.

PROPOSITION 6.3. Let $\left\{K_{n}\right\}_{n \geq 1}$ be a generator for some f-register-shift $R$. Then $\left\{K_{n}\right\}_{n \geq 1}$ is splitting.

Proof. Assume that $\left\{K_{n}\right\}_{n \geq 1}$ is not splitting. Then there exists $m \geq 1$ and a component $B$ of $K_{m}$ such that for all $n \geq m, B$ contains exactly one component of $K_{n}$ (since 
by lemma 4.1(1) $B$ contains at least one component of $K_{n}$ ). For $n \geq m$ let $B_{n}$ be the unique component of $K_{n}$ with $B_{n} \subseteq B$. Then $B_{n+1} \subseteq B_{n}$ and card $\left(\bigcap_{n \geq m} B_{n}\right)=1$. Without loss of generality we can assume that $S\left(f, K_{n}\right)=S\left(f, K_{m}\right)$ for all $n \geq m$. By proposition $4.4(2)$ we have $S\left(f, K_{m}\right) \neq \varnothing$, and thus there exists $(x, \alpha) \in I^{*}$ with $(x, \alpha) \in S\left(f, K_{m}\right) \subseteq\left(K_{n}\right)^{*}$ for all $n \geq 1$. Lemma 6.2 shows that card $\left(O_{f}(x, \alpha)\right)=+\infty$; hence there exist $c, d \in O_{f}(x, \alpha) \subseteq O_{f}\left(S\left(f, K_{m}\right)\right)$ with $c<d$ such that $(c, d) \subseteq K_{m}$. We can find $p \geq 0$ such that $f^{p}((c, d)) \cap B \neq \varnothing$. By lemma 6.1 we have

$$
\begin{aligned}
\partial\left(f^{p}((c, d))\right) & \subseteq O_{f}\left(S\left(f, K_{m}\right)\right) \cup\left\{f^{p}(c, 1), f^{p}(d,-1)\right\} \\
& \subseteq O_{f}\left(S\left(f, K_{m}\right)\right) ;
\end{aligned}
$$

thus there exist $u, v \in O_{f}\left(S\left(f, K_{m}\right)\right)$ with $(u, v) \subseteq B$. But since $O_{f}\left(S\left(f, K_{m}\right)\right) \subseteq K_{n}$ for all $n \geq 1$ and $B$ is not $\left\{K_{n}\right\}_{n \geq 1}$-splitting, it follows that $(u, v) \subseteq B_{n}$ for all $n \geq m$ which is not possible.

Note that by the above proposition each point of an $f$-register-shift $R$ is a limit point of $R$. Since in addition $R$ is non-empty, closed and nowhere dense, $R$ is a Cantor-like set. In particular, for each $x \in R$ there exists $\alpha \in\{1,-1\}$ with $(x, \alpha) \in R^{*}$. The next proposition shows that $f$ acts minimally on each $f$-register-shift.

Proposition 6.4. Let $R$ be an f-register-shift. Then $\overline{O_{f}(x, \alpha)}=R$ for each $(x, \alpha) \in R^{*}$. Proof. Let $(x, \alpha) \in R^{*},\left\{K_{n}\right\}_{n \geq 1}$ be a generator for $R$ and for each $n \geq 1$ and $(\varphi, \beta) \in S\left(f, K_{n}\right)$ let $K_{n}(\varphi, \beta)$ denote the unique component of int $\left(K_{n}\right)-S(f)$ with $(\varphi, \beta) \in\left(K_{n}(\varphi, \beta)\right)^{*}$. We can find $m \geq 1$ such that for each $(\varphi, \beta) \in S\left(f, K_{m}\right)$ either

$$
O_{f}(x, \alpha) \cap\left(\overline{K_{m}(\varphi, \beta)}-\{\varphi\}\right)=\varnothing
$$

or

$$
O_{f}(x, \alpha) \cap K_{n}(\varphi, \beta) \neq \varnothing
$$

for all $n \geq m$. Now let $z \in R$ and for $n \geq m$ let $B_{n}$ denote the unique component of $K_{n}$ with $z \in B_{n}$. Then $\{z\}=\bigcap_{n \geq m} B_{n}$. Since $(x, \alpha) \in\left(K_{n}\right)^{*}$ we have $O_{f}(x, \alpha) \subseteq K_{n}$ for each $n \geq 1$ and thus $O_{f}(x, \alpha) \subseteq R$. Hence it is sufficient to show that $O_{f}(x, \alpha) \cap B_{n} \neq \varnothing$ for all $n \geq m$. So let $n \geq m$. Since $(x, \alpha) \in R^{*} \subseteq\left(K_{j}\right)^{*}$ for all $j \geq 1$, lemma 6.2 shows that there exist $c, d \in O_{f}(x, \alpha)$ with $c<d$ and $(c, d) \subseteq K_{n}$. Moreover, we can find $p \geq 0$ such that

$$
f^{p}\left((c, d)-S\left(f^{p}\right)\right) \cap B_{n} \neq \varnothing .
$$

Hence $O_{f}(x, \alpha) \cap B_{n} \neq \varnothing$ follows if we can show that $\partial\left(f^{p}\left((c, d)-S\left(f^{p}\right)\right)\right) \subseteq \overline{O_{f}(x, \alpha)}$. Let $u, v \in \overline{O_{f}(x, \alpha)}$ with $(u, v) \subseteq K_{n}$ and let $w \in \partial(f((u, v)-S(f)))$. Then either $w=$ $f(u), w=f(v)$ or $w=f(\varphi, \beta)$ for some $(\varphi, b) \in S\left(f, K_{n}\right)$. If $w=f(u)$ or $w=f(v)$ then clearly $w \in \overline{O_{f}(x, \alpha)}$. If $w=f(\varphi, \beta)$ for some $(\varphi, \beta) \in S\left(f, K_{n}\right)$ then by the choice of $m$ we have $K_{j}(\varphi, \beta) \cap O_{f}(x, \alpha) \neq \varnothing$ for all $j \geq m$; hence $w \in \overline{O_{f}(x, \alpha)}$ (since $\left.\bigcap_{j \geq m} \overline{K_{j}(\varphi, \beta)}=\{\varphi\}\right)$. Repeating this argument shows that $\partial\left(f^{k}\left((u, v)-S\left(f^{k}\right)\right)\right) \subseteq$ $\overline{O_{f}(x, \alpha)}$ for all $k \geq 0$; in particular, we have

$$
\partial\left(f^{p}\left((c, d)-S\left(f^{p}\right)\right)\right) \subseteq \overline{O_{f}(x, \alpha)}
$$

Let $R$ be an $f$-register-shift; the above result gives us that $O_{f}(x)$ is dense in $R$ for all $x \in R-M(f)$ (note that since $M(f)$ is countable and $R$ is a Cantor-like set, 
$R-M(f)$ is uncountable). Next we will show that $R$ is the union of $f(R-S(f))$ and a finite set. For this we need the following lemma which will also be useful later.

LemMA 6.5. Let $A \subseteq I$ be closed and let $(y, \beta) \in(\overline{f(A-S(f)})^{*}$. Then there exists $(x, \alpha) \in A^{*}$ with $F(x, \alpha)=(y, \beta)$.

Proof. For each $n \geq 1$ there exists $y_{n} \in f(A-S(f)) \cap B_{1 / n}(y, \beta)$. For $n \geq 1$ let $x_{n} \in A$ with $f\left(x_{n}\right)=y_{n}$ and let $x$ be a point of accumulation of $\left\{x_{n}\right\}_{n \geq 1}$. Then there exists $\alpha \in\{1,-1\}$ such that $B_{\varepsilon}(x, \alpha) \cap\left\{x_{n}: n \geq 1\right\} \neq \varnothing$ for each $\varepsilon>0$, and so $(x, \alpha) \in A^{*}$ and $F(x, \alpha)=(y, \beta)$.

Proposition 6.6. Let $R$ be an f-register-shift. Then:

(1) $\overline{f(R-S(f))}=R$.

(2) $f(R-S(f)) \cup\left\{f(x, \alpha):(x, \alpha) \in R^{*}\right.$ and $\left.x \in S(f)\right\}=R$.

(3) $F\left(R^{*}\right)=R^{*}$.

Proof. (1) Let $\left\{K_{n}\right\}_{n \geq 1}$ be a generator for $R$. Then

$$
f(R-S(f)) \subseteq f\left(K_{n}-S(f)\right) \subseteq K_{n} \quad \text { for each } n \geq 1 ;
$$

hence $f(R-S(f)) \subseteq R$. Let $x \in R-M(f)$; then $O_{f}(f(x)) \subseteq f(R-S(f)) \subseteq R$ and thus by proposition 6.4 we have $\overline{f(R-S(f))}=R$.

(2) Clearly by (1) we have

$$
f(R-S(f)) \cup\left\{f(x, \alpha):(x, \alpha) \in R^{*} \text { and } x \in S(f)\right\} \subseteq \overline{f(R-S(f)}=R .
$$

Now let $y \in R-f(R-S(f))$; then $\left.(y, \beta) \in R^{*}=\overline{(f(R-S(f))}\right)^{*}$ for some $\beta \in\{1,-1\}$, and so by lemma 6.5 there exists $(x, \alpha) \in R^{*}$ with $f(x, \alpha)=y$. Since $y \notin f(R-S(f))$ we must have $x \in S(f)$.

(3) Let $(x, \alpha) \in R^{*}$; then $f(x, \alpha) \in R$ and for all $\varepsilon>0$ we have $B_{\varepsilon}(f(x, \alpha), \Pi(x, \alpha)) \cap R \neq \varnothing$. Hence $F(x, \alpha) \in R^{*}$. On the other hand, let $(y, \beta) \in R^{*}$. Then by $(1)(y, \beta) \in(\overline{f(R-S(f))})^{*}$ and so by lemma 6.5 there exists $(x, \alpha) \in R^{*}$ with $F(x, \alpha)=(y, \beta)$.

Let $A \subseteq I$ be non-empty; for $x \in I$ put $d(x, A)=\inf \{|x-y|: y \in A\}$.

Let $R$ be an $f$-register-shift; the next result shows that each element of $A(R, f)$ is attracted to $R$.

Proposition 6.7. Let $R$ be an $f$-register-shift and $\left\{K_{n}\right\}_{n \geq 1}$ be a generator for $R$. Then:

(1) For each $\varepsilon>0$ there exists $m \geq 1$ such that if $x \in A\left(K_{m}, f\right)$ and $\alpha \in\{1,-1\}$ then $\lim \sup _{n \rightarrow \infty} d\left(f^{n}(x, \alpha), R\right) \leq \varepsilon$.

(2) If $x \in A(R, f)$ and $\alpha \in\{1,-1\}$ then $\lim _{n \rightarrow \infty} d\left(f^{n}(x, \alpha), R\right)=0$.

Proof. (1) Let $\varepsilon>0$; there exists $m \geq 1$ such that the length of each component of $K_{m}$ is smaller than $\varepsilon$ and

$$
\text { int }\left(K_{m}\right) \cap S(f)=\operatorname{int}\left(K_{n}\right) \cap S(f) \quad \text { for all } n \geq m \text {. }
$$

Let $x \in A\left(K_{m}, f\right)$ and $\alpha \in\{1,-1\}$. If $x \in A\left(K_{m}, f\right) \cap M(f)$ then there exists $p \geq 0$ such that $f^{p}(x) \in$ int $\left(K_{m}\right) \cap S(f) \subseteq R$; thus $f^{n}(x, \alpha) \in R$ for all $n \geq p$.

If $x \in A\left(K_{m}, f\right)-M(f)$ then there exists $p \geq 0$ such that $f^{n}(x) \in K_{m}$ for all $n \geq p$. Hence in any case $\lim \sup _{n \rightarrow \infty} d\left(f^{n}(x, \alpha), R\right) \leq \varepsilon$.

(2) This follows immediately from (1). 
Let $R$ be an $f$-register-shift; one can show that if $x \in I-M(f)$ with $\lim _{n \rightarrow \infty} d\left(f^{n}(x), R\right)=0$ then it does not necessarily follow that $x \in A(R, f)$.

Let $C$ be an $f$-cycle; we call $C$ strongly transitive if for each non-empty open subset $U$ of $C$ we have $\bigcup_{n=0}^{m} \overline{f^{n}\left(U-S\left(f^{n}\right)\right)}=C$ for some $m \geqslant 0$.

In order to prove that each topologically transitive $f$-cycle is strongly transitive we need the following result.

Proposition 6.8. Let $\varphi \in S(f)$ and suppose that $\overline{L_{m}(\varphi)}$ is a topologically transitive $f$-cycle for some $m \geq 1$. Then there exists $n \geq 0$ such that

$$
L_{m}(\varphi)=\bigcup_{k=1}^{n} f^{k}\left(I_{m}(\varphi)-S\left(f^{k}\right)\right) .
$$

Proof. By proposition 4.3 we have $L_{m}(\varphi) \subseteq \overline{M(f)}$; hence by lemma 5.3, $L_{m}(\varphi)=$ $\left(c_{1}, d_{1}\right) \cup \cdots \cup\left(c_{q}, d_{q}\right)$ can be written as a disjoint and finite union of non-empty open intervals. Put $B(1)=\left\{c_{1}, \ldots, c_{q}\right\}$ and $B(-1)=\left\{d_{1}, \ldots, d_{q}\right\}$. For $n \geq 0$ let

$$
E_{n}=\bigcup_{0 \leq k \leq n} f^{k}\left(I_{m}(\varphi)-S\left(f^{k}\right)\right)
$$

then $L_{m}(\varphi)=\bigcup_{n \geq 0} E_{n}, E_{n} \subseteq E_{n+1}$ and $f^{j}\left(E_{n}-S\left(f^{j}\right)\right) \subseteq E_{n+j}$ for all $n, j \geq 0$. For $\alpha \in$ $\{1,-1\}$ put

$$
R(\alpha)=\left\{z \in B(\alpha):(z, \alpha) \notin\left(\overline{E_{n}}\right)^{*} \text { for all } n \geq 0\right\} .
$$

Now let us assume that $L_{m}(\varphi) \neq E_{n}$ for all $n \geq 0$. Then $R(1) \cup R(-1) \neq \varnothing$. Let $\varepsilon>0$; for $\alpha \in\{1,-1\}$ and $u \in B(\alpha)$ put $u(\varepsilon)=u+\alpha \cdot \varepsilon$ if $u \in R(\alpha)$ and $u(\varepsilon)=u$ if $u \notin R(\alpha)$. Furthermore, let $D(\varepsilon)=\bigcup_{1 \leq i \leq q}\left(c_{i}(\varepsilon), d_{i}(\varepsilon)\right)$. By compactness there exists $k \geq 0$ such that $\bigcup_{1 \leq i \leq q}\left(c_{i}+\varepsilon, d_{i}-\varepsilon\right) \subseteq E_{k}$. Hence there exists $j \geq 0$ such that $D(\varepsilon) \subseteq E_{j}$.

We will first show that for each $u \in R(\alpha)$ there exists $j \geq 1$ such that $F^{j}(u, \alpha)=$ $(u, \alpha)$. Let $u \in R(\alpha)$ for some $\alpha \in\{1,-1\}$. Since $\overline{L_{m}(\varphi)}$ is a topologically transitive $f$-cycle we have

$$
\overline{f\left(\overline{L_{m}(\varphi)}-\overline{S(f))}\right.}=\overline{L_{m}(\varphi)}
$$

hence by lemma 6.5 there exists $\left(u_{1}, \alpha_{1}\right) \in\left(\overline{L_{m}(\varphi)}\right)^{*}$ with $F\left(u_{1}, \alpha_{1}\right)=(u, \alpha)$. Then $\left(u_{1}, \alpha_{1}\right) \notin\left(\overline{E_{n}}\right) *$ for all $n \geq 0$ and thus $u_{1} \in R\left(\alpha_{1}\right)$. Therefore, we can inductively find a sequence $\left\{\left(u_{n}, \alpha_{n}\right)\right\}_{n \geq 0}$ of elements of $\left(\overline{L_{m}(\varphi)}\right)^{*}$ such that $\left(u_{0}, \alpha_{0}\right)=(u, \alpha)$, $u_{n} \in R\left(\alpha_{n}\right)$ and $F\left(u_{n+1}, \alpha_{n+1}\right)=\left(u_{n}, \alpha_{n}\right)$ for each $n \geq 0$. Since card $(R(1) \cup R(-1))<+\infty$ there exist $n \geq 0$ and $j \geq 1$ such that $\left(u_{j+n}, \alpha_{j+n}\right)=\left(u_{n}, \alpha_{n}\right)$. Hence $\left(u_{j}, \alpha_{j}\right)=\left(u_{0}, \alpha_{0}\right)$ and thus $F^{l j}(u, \alpha)=(u, \alpha)$ for each $l \geq 0$. Since by proposition $4.3(1)$ int $\left(\overline{L_{m}(\varphi)}\right) \cap \operatorname{Sink}(f)=\varnothing$ there exist $N \geq 1$ and $\varepsilon>0$ such that

(i) for each $v \in R(\beta), \beta \in\{1,-1\}, f^{N}$ is continuous and increasing on $B_{\varepsilon}(v, \beta), F^{N}(v, \beta)=(v, \beta)$ and $\beta \cdot f^{N}(z)>\beta \cdot z$ for all $z \in B_{\varepsilon}(v, \beta)$

(ii) $I_{m}(\varphi) \cup\left(L_{m}(\varphi) \cap S\left(f^{N}\right)\right) \subseteq D(\varepsilon)$.

Furthermore, there exist $p, k \geq 1$ and $0<\delta<\varepsilon$ such that

$$
D(\varepsilon) \subseteq E_{k} \subseteq E_{k+N} \subseteq D(\delta) \subseteq E_{p} .
$$

If $f^{N}\left(D(\delta)-S\left(f^{N}\right)\right) \subseteq D(\delta)$ then (since $I_{m}(\varphi) \subseteq D(\delta)$ ) we would have $L_{m}(\varphi) \subseteq$ $\bigcup_{i=0}^{N-1} f^{i}\left(D(\delta)-S\left(f^{i}\right)\right) \subseteq E_{p+N-1}$ which contradicts our assumption. Hence there exists $x \in D(\delta)$ with $f^{N}(x) \in L_{m}(\varphi)-D(\delta)$. Without loss of generality let us assume 
that $f^{N}$ is increasing in $x$ and that $f^{N}(x) \in(c, c+\delta]$ for some $c \in R(1)$. Put

$$
y=\max \left\{z \leq x: z \in S\left(f^{N}\right) \cup B(1)\right\} ;
$$

so $y<x$ and $f^{N}((y, x)) \subseteq(c, c+\delta)$. If $y \in S\left(f^{N}\right)$ or $y \in B(1)-R(1)$ then we would have $(y, x) \cap E_{k} \neq \varnothing$ and thus $f^{N}((y, x)) \cap E_{k+N} \neq \varnothing$; but this is not possible since $E_{k+N} \cap(c, c+\delta)=\varnothing$. Hence $y \in R(1)$. Since $x \in D(\delta)$ we have $B_{\delta}(y, 1) \subseteq(y, x)$ and so $f^{N}\left(B_{\delta}(y, 1)\right) \subseteq(c, c+\delta)$. But again this is not possible since by (i) the length of the interval $f^{N}\left(B_{\delta}(y, 1)\right)$ is strictly larger than $\delta$. Therefore, $L_{m}(\varphi)=E_{n}$ for some $n \geq 0$.

Proposition 6.9. $C$ is a topologically transitive $f$-cycle if and only if $C$ is a strongly transitive $f$-cycle.

Proof. If $C$ is a strongly transitive $f$-cycle then clearly, by proposition $4.2, C$ is topologically transitive. Conversely, suppose that $C$ is a topologically transitive $f$-cycle. By theorem 5.9 there exist $\varphi \in S(f)$ with int $(\overline{\Delta(\varphi)}) \neq \varnothing$ and $m \geq 1$ such that $C=\overline{L_{n}(\varphi)}$ for all $n \geq m$. Now let $U \subseteq C$ be non-empty and open. Since $U \subseteq C \subseteq \overline{\Delta(\varphi)}$ we have $I_{j}(\varphi) \subseteq f^{q}\left(U-S\left(f^{q}\right)\right)$ for some $q \geq 0$ and $j \geq m$. Hence by proposition 6.8 there exists $p \geq q$ such that

$$
L_{j}(\varphi) \subseteq \bigcup_{k=0}^{p} f^{k}\left(U-S\left(f^{k}\right)\right)
$$

Since $\overline{L_{j}(\varphi)}=C$ this shows that $C$ is strongly transitive.

Remark. We call $(x, \alpha) \in I^{*}$ periodic if $F^{n}(x, \alpha)=(x, \alpha)$ for some $n \geq 1$. We say that $g \in \mathcal{N}(I)$ is uniformly piecewise linear with slope $\eta>0$ if on each component $U$ of $I-S(f) g$ is linear with slope $\eta$ or $-\eta$. Suppose that $I$ is a strongly transitive $f$-cycle, and assume further that either $f(U) \cap f(V) \neq \varnothing$ for some components $U$ and $V$ of $I-S(f)$ with $U \neq V$ or that $I^{*}$ contains no periodic element. As in [13, Theorem $5]$ it can be shown that $f$ is then conjugate to a uniformly piecewise linear mapping $g \in \mathcal{N}(I)$ (with slope $\eta \geq 1$ ), i.e. there exists a homeomorphism $\psi$ of $I^{\prime}$ such that $\psi f=g \psi$.

Let $C$ be an $f$-cycle and assume that there exists a continuous function $g$ : $C \rightarrow C$ such that $f(x)=g(x)$ for all $x \in C-S(f)$. Then we can label the components $B_{1}, \ldots, B_{m}$ of $C$ in such a way that $g\left(B_{i}\right) \subseteq B_{i+1}$ for $1 \leq i \leq m-1$ and $g\left(B_{m}\right) \subseteq B_{1}$. For $n \geq 0$ define inductively $g^{0}(x)=x$ and $g^{n+1}(x)=g\left(g^{n}(x)\right)$ for all $x \in C$. Let $1 \leq i \leq m$; then $g^{m}\left(B_{i}\right) \subseteq B_{i}$, and if in addition $C$ is strongly transitive then for each non-empty open subset $U \subseteq B_{i}$ there exists $p \geq 0$ such that $\bigcup_{k=0}^{p} g^{m k}(U)=B_{i}$. The next result shows that in this situation proposition 6.9 can be improved.

Proposition 6.10. Let $C$ be a topologically transitive $f$-cycle with $m$ components, let $B$ be a component of $C$ and suppose that there exists a continuous function $g: C \rightarrow C$ such that $g(x)=f(x)$ for all $x \in C-S(f)$. Then exactly one of the following statements holds:

(1) For each non-trivial interval $J \subseteq B$ there exists $k \geq 0$ with $\mathrm{g}^{\mathrm{km}}(J)=B$.

(2) There exists a closed interval $D$ with $D \cup g^{m}(D)=B$, int $(D) \cap \operatorname{int}\left(g^{m}(D)\right)=\varnothing$ and $g^{2 m}(D)=D$ such that for each non-trivial interval $J \subseteq D$ there exists $k \geq 0$ with $g^{k m}(J)=D$. 
Proof. Put $B=[c, d]$ and $h=g^{m}$. By Proposition 6.9, $C$ is strongly transitive; in particular, we have $h(B)=B$ and $h(u)=u$ for some $u \in(c, d)$. Suppose that there exists a non-trivial interval $J \subseteq B$ such that $h^{n}(J) \neq B$ for all $n \geq 0$. Since $C$ is strongly transitive there exists $j \geq 0$ such that $u \in h^{n}(J)$ for all $n \geq j$; hence $[c, u] \subseteq$ $h^{p}(J)$ for some $p \geq j$. Since $h^{n}(J) \neq B$ for all $n \geq 0$ we have $c \notin h([c, u])$, and thus $c \in h([u, d])$. The same argument shows that $d \in h([c, u])$. Therefore, for all $n \geq 0$ we have

$$
[c, u] \subseteq h^{2 n}([c, u]) \subseteq h^{p+2 n}(J) \subseteq[c, d)
$$

and

$$
[u, d] \subseteq h^{2 n+1}([c, u]) \subseteq h^{p+2 n+1}(J) \subseteq(c, d]
$$

For $n \geq 0$ put

$$
D_{n}=h^{p+2 n}(J) \cap h^{p+2 n+1}(J) .
$$

Then $D_{n}$ is an interval with $u \in D_{n}$ and $h\left(D_{n}\right) \subseteq D_{n+1} \subseteq(c, d)$ for each $n \geq 0$. Hence $D_{n}=\{u\}$ for all $n \geq 0$ because $C$ is strongly transitive. Therefore, putting $D=[c, u]$ we have $h(D)=[u, d]$ and $h^{2}(D)=D$. Now let $J^{\prime} \subseteq D$ be a non-trivial interval; as above we have $[c, u] \subseteq h^{k}\left(J^{\prime}\right)$ for some $k \geq 0$, and thus $[c, u] \subseteq h^{k}\left(J^{\prime}\right) \subseteq h^{k}(D)=$ $[c, u]$. Finally, it is clear that (1) and (2) cannot both hold.

\section{Some examples}

In this section we want to apply our results to some examples. We will make use of the following fact.

Proposition 7.1. Suppose that Sink $(f)=\operatorname{Homt}(f)=\varnothing$ and that $I$ is the only $f$-cycle $K$ with int $(K) \cap S(f) \cap(a, b) \neq \varnothing$. Then $I$ is strongly transitive.

Proof. By proposition 4.4(2) there are no $f$-register-shifts. Thus by theorem 5.1 there exists a topologically transitive $f$-cycle $K$. We have $K=I$ since by proposition $4.3(3)$ int $(K) \cap S(f) \cap(a, b) \neq \varnothing$, and by proposition $6.9 I$ is strongly transitive.

Suppose that $g \in \mathcal{N}([0,1])$ is given by $g(x)=\beta x \bmod 1$ for all $x \in(0,1)$ with $\beta x \notin \mathbb{N}$ and some $\beta>1$ (such transformations were discussed in [17]). Then $I$ is a strongly transitive $g$-cycle; this follows immediately from the next corollary.

For an interval $J \subseteq I$ let $|J|$ denote the length of $J$.

COROllary 7.2. Suppose that $f(a, 1)=a, f(\varphi, 1)=a$ and $f(\varphi,-1)=b$ for all $\varphi \in S(f) \cap(a, b)$ and that $|f(J)|>|J|$ for each interval $J \subseteq I$ with $J \cap S(f)=\varnothing$. Then $I$ is a strongly transitive $f$-cycle.

Proof. Since $|f(J)|>|J|$ for each interval $J \subseteq I$ with $J \cap S(f)=\varnothing$ we clearly have Sink $(f)=$ Homt $(f)=\varnothing$. Let $K$ be an $f$-cycle with int $(K) \cap S(f) \cap(a, b) \neq \varnothing$. Then $f(\varphi, 1)=a$ for each $\varphi \in S(f) \cap(a, b)$ implies that $a \in K$. Since $f(a, 1)=a, f(\varphi,-1)=b$ for each $\varphi \in S(f) \cap(a, b)$ and Sink, $(f)=\varnothing$ it follows that $K=I$. Therefore, by proposition 7.1, $I$ is strongly transitive.

The next class of function we consider contains the Poincaré map of the geometric Lorentz attractor already mentioned in the Introduction. 
COROLlaRY 7.3. Suppose that $f$ is continuous and strictly increasing on $(a, \varphi)$ and $(\varphi, b)$ for some $a<\varphi<b$, that $f(\varphi, 1)=a$ and $f(\varphi,-1)=b$ and that $|f(J)|>\sqrt{2}|J|$ for each interval $J \subseteq I$ with $J \cap S(f)=\varnothing$. Then $I$ is a strongly transitive $f$-cycle.

Proof. Since $|f(J)|>\sqrt{2}|J|$ for each interval $J \subseteq I$ with $J \cap S(f)=\varnothing$ we have Sink $(f)=$ Homt $(f)=\varnothing$. Let $K$ be an $f$-cycle with $\varphi \in$ int $(K) \cap S(f) \cap(a, b)$ and let $B=[c, d]$ be the component of $K$ with $\varphi \in B$. Put $U_{1}=(c, \varphi)$ and $U_{2}=(\varphi, d)$. Without loss of generality we can assume that $\left|U_{1}\right| \geq\left|U_{2}\right|$ (otherwise 'turn $f$ upside down'). Put $n=\min \left\{k \geq 1: f^{k}\left(U_{1}\right) \cap B \neq \varnothing\right\}$. Then $f^{n}\left(U_{1}\right) \subseteq B$, and we have

$$
|B|>(\sqrt{2})^{n} \cdot\left|U_{1}\right| \geq \frac{1}{2}(\sqrt{2})^{n} \cdot|B| ;
$$

hence $n=1$ and $d=b$ (because $f(\varphi,-1)=b)$. Moreover, we have $\sqrt{2}(\varphi-a)<b-a$; thus

$$
\left|U_{2}\right|>b-\left(\frac{1}{2} \sqrt{2}(b-a)+a\right)=\left(1-\frac{1}{2} \sqrt{2}\right)(b-a)
$$

and

$$
\left|f\left(U_{2}\right)\right|>(\sqrt{2}-1)(b-a) .
$$

Therefore, $|B|+\left|f\left(U_{2}\right)\right|>b-a$ which gives us that $f\left(U_{2}\right) \cap B \neq \varnothing$ and thus $f\left(U_{2}\right) \subseteq B$. Hence $B$ is the only component of $K$. Since $f(\varphi, 1)=a$ we have $c=a$ and therefore $K=I$. Thus, by proposition $7.1, I$ is strongly transitive.

Finally we will apply our results to interval exchange transformations (see for instance $[4]$ or $[8]$ ).

Let $S(f)=\left\{d_{0},,,, d_{m+1}\right\}$ with $a=d_{0}<d_{1}<\cdots<d_{m+1}=b . f$ is said to be an interval exchange transformation (on $I$ ) if $f$ 'exchanges' the open intervals $\left(d_{k}, d_{k+1}\right), k=0, \ldots, m$ according to a permutation of $\{0,1, \ldots, m\}$, i.e. if

(7.1) $f$ is linear with slope 1 on each of the open intervals $\left(d_{k}, d_{k+1}\right), k=0, \ldots, m$; and

(7.2) $f\left(\left(d_{k}, d_{k+1}\right)\right) \cap f\left(\left(d_{j}, d_{j+1}\right)\right)=\varnothing$ if $0 \leq k \leq j \leq m$.

Now suppose that $f$ is an interval exchange transformation. Then $F^{n}(x, 1)=\left(f^{n}(x, 1), 1\right)$ for all $n \geq 1, x \in[a, b), F: I^{*} \rightarrow I^{*}$ is bijective and

$$
f(I-S(f)) \cup\{(\varphi, 1): \varphi \in S(f)-\{b\}\}=[a, b) .
$$

Moreover, $f^{n}$ is also an interval exchange transformation for each $n \geq 1$, and it is not difficult to see that for each $f$-cycle $K$ we have $f^{-1}(K) \subseteq K$; in particular, this gives us that $A(K, f) \subseteq K$.

Proposition 7.4. Suppose that $f$ is an interval exchange transformation. Then:

(1) If $x \in I$ with $f^{n}(x)=x$ for some $n \geq 1$ then $x$ is contained in some sink of $f$.

(2) Sink $(f)=\left\{x \in I\right.$ : there exists $n \geq 1$ and $u, v \in S\left(f^{n}\right)$ with $u<x<v$ such that $f^{n}(z)=z$ for all $\left.z \in(u, v)\right\}$.

(3) $\operatorname{Homt}(f)=\varnothing$.

Proof. (1) Suppose that $x \in I$ with $f^{n}(x)=x$ for some $n \geq 1$. Then there exist $u, v \in S\left(f^{n}\right)$ with $u<x<v$ and $(u, v) \cap S\left(f^{n}\right)=\varnothing$. Since $f^{n}$ is linear on $(u, v)$ with slope 1 we have $f^{n}(z)=z$ for all $z \in(u, v)$. Hence $(u, v)$ is a sink of $f$ with $x \in(u, v)$. 
(2) Clearly, if $u, v \in S\left(f^{n}\right)$ with $n \geq 1, u<v$ and $f^{n}(z)=z$ for all $z \in(u, v)$ then $(u, v)$ is a sink of $f$ and thus $(u, v) \subseteq \operatorname{Sink}(f)$. Conversely, let $J$ be a sink of $f$ and let $n \geq 1$ with $f^{n}(J) \subseteq J$. Then there exist $u, v \in S\left(f^{n}\right)$ with $J \subseteq(u, v)$ and $(u, v) \cap S\left(f^{n}\right)=\varnothing$. Since $f^{n}$ is linear on $(u, v)$ with slope 1 we have $f^{n}(z)=z$ for all $z \in(u, v)$. Moreover, since $f^{n}(J)=J$ it follows that

$$
\left\{x \in I: f^{n}(x) \in J \text { for some } n \geq 0\right\}=\bigcup_{k=0}^{n-1} f^{k}(J),
$$

and thus each element of Sink $(f)$ is already contained in a sink of $f$.

(3) This follows immediately from Proposition 3.3(3) and from the fact that if $J \subseteq I$ is an interval with $J \cap S(f)=\varnothing$ then $|J|=|f(J)|$.

COROLlaRy 7.5. Suppose that $f$ is an interval exchange transformation and let $C_{1}, \ldots, C_{r}$ be the topologically transitive $f$-cycles. Then:

(1) There are no f-register-shifts.

(2) $\operatorname{Sink}(f) \cup C_{1} \cup \cdots \cup C_{r}$ is dense in $I$.

Proof. (1) Let $R$ be an $f$-register-shift. By theorem 5.9 and proposition 5.6 there exists $\varphi \in S(f)$ with int $\overline{\Delta(\varphi)} \neq \varnothing$ and $m \geq 1$ such that $R=\bigcap_{n \geq 1} \overline{L_{n}(\varphi)}$ and $\overline{L_{n}(\varphi)} \subseteq$ $\overline{\Delta(\varphi)}$ for all $n \geq m$. But since $f^{-1}\left(\overline{L_{n}(\varphi)}\right) \subseteq \overline{L_{n}(\varphi)}$ we have $\Delta(\varphi) \subseteq \overline{L_{n}(\varphi)}$ and thus $\overline{L_{n}(\varphi)}=\overline{\Delta(\varphi)}$ for each $n \geq m$. This is not possible since int $(R)=\varnothing$.

(2) Since $A(K, f) \subseteq K$ for each $f$-cycle $K$ and since by proposition 7.4(3), Homt $(f)=\varnothing$ this follows immediately from theorem 5.1.

Finally, we show that if the orbits $O_{f}(\varphi, 1)$ of the singular points $\varphi$ in $(a, b)$ are infinite and disjoint then $I$ is a strongly transitive $f$-cycle.

COROllary 7.6 (cf. [8].) Suppose that $f$ is an interval exchange transformation with $S(f) \cap(a, b) \neq \varnothing$ and assume that card $\left(O_{f}(\varphi, 1)\right)=+\infty$ and $f^{n}(\varphi, 1) \notin S(f) \cap(a, b)$ for all $\varphi \in S(f) \cap(a, b)$ and $n \geq 1$. Then I is a topologically transitive f-cycle. Moreover, we have $O_{f}(x, 1)$ is dense in I for each $x \in[a, b)$.

Proof. We will first show that $\operatorname{Sink}(f)=\varnothing$. Assume that $\operatorname{Sink}(f) \neq \varnothing$. By proposition $7.4(2)$ there exist $n \geq 1$ and $u, v \in S\left(f^{n}\right)$ with $u<v$ such that $f^{n}(z)=z$ for all $z \in(u, v)$; then $f^{k}(u) \in S(f)$ for some $0 \leq k<n$, Put $\varphi=f^{k}(u)$; so $\varphi<b$ and $f^{n}(\varphi, 1)=\varphi$. Thus by assumption we have $\varphi=a$. Since $a=f(\eta, 1)$ for some unique $\eta \in S(f) \cap[a, b)$ it follows that $f^{n}(\eta, 1)=\eta$. But again this is only possible if $\eta=a$; hence $f(a, 1)=a$, and thus $f(z)=z$ for all $z \in(a, \xi)$ where $\xi=\min (S(f) \cap(a, b))$. But then there exists $\psi \in S(f) \cap[\xi, b)$ with $f(\psi, 1)=\xi$, which contradicts our assumption. Therefore, $\operatorname{Sink}(f)=\varnothing$.

Let $C_{1}, \ldots, C_{r}$ be the topologically transitive $f$-cycles. Then by corollary 7.5(2) $I=$ $C_{1} \cup \cdots \cup C_{r}$. Assume that $r \geq 2$. Then $C_{1} \cap C_{j} \neq \varnothing$ for some $1 \leq i<j \leq r$. Let $z \in C_{i} \cap$ $C_{j}$. Suppose first that $z \notin M(f)$. Then $f^{n}(z) \in C_{i} \cap C_{j}$ for all $n \geq 0$, and thus there would exist $p \geq 1$ and $m \geq 0$ such that $f^{p}\left(f^{n}(z)\right)=f^{n}(z)$; by proposition 7.4(1) this is not possible. Suppose next that $z \in M(f)$; then $f^{m}(z) \in S(f) \cap(a, b)$ for some $m \geq 0$ and there exists $(x, 1) \in I^{*}$ with $f(x, 1)=z$. Since $f^{n}(\varphi, 1) \notin S(f) \cap(a, b)$ for all $\varphi \in S(f) \cap[a, b)$ and $n \geq 1\left(f^{n}(a, 1) \notin S(f) \cap(a, b)\right.$ for all $n \geq 1$ because $a=f(\xi, 1)$ for some $\xi \in S(f) \cap(a, b))$ it follows that $x \notin S(f)$. Thus $x \in f^{-1}\left(C_{i} \cap C_{j}\right) \subseteq C_{i} \cap C_{j}$. 
Repeating this argument shows that there exists $p \geq 1$ with $f^{p}(x)=x$ which by proposition 7.4(1) is not possible. Hence $r=1$.

Let $x \in[a, b)$ and let $J \subseteq I$ be a non-trivial closed interval. By proposition $6.9 I$ is strongly transitive; thus we have $I=\bigcup_{n=0}^{m} \overline{f^{n}\left(J-S\left(f^{n}\right)\right)}$ for some $m \geq 0$. Let $z=f^{m}(x, 1)$. It follows from lemma 6.5 that there exist $y \in J$ and $0 \leq k \leq m$ such that $f^{k}(y, 1)=z$. But this immediately gives us that $f^{m-k}(x, 1)=y \in J$. Therefore, $O_{f}(x, 1)$ is dense in $I$.

\section{REFERENCES}

[1] B. Barna. Über die Divergenzpunkte des Newtonschen Verfahrens zur Bestimmung von Wurzeln algebraischer Gleichungen, III. Publ. Math. Debrecen 8 (1961), 193-207.

[2] P. Collet \& J.-P. Eckmann. Iterated Maps on the Interval as Dynamical Systems. Progress in Physics, Vol. 1, Birkhäuser: Boston, 1980.

[3] P. Collet. J.-P. Eckmann \& O. E. Lanford, III. Universal properties of maps on an interval. Commun. Math. Phys. 76 (1980), 211-254.

[4] I. P. Cornfeld, S. V. Fomin \& Ya. G. Sinai. Ergodic Theory. Springer: New York, 1982.

[5] E. M. Coven \& Z. Nitecki. Non-wandering sets of the powers of maps of the interval. Ergod. Th. \& Dynam. Sys. 1 (1981), 9-31.

[6] M. Feigenbaum. Quantitative universality for a class of non-linear transformations. J. Stat. Phys. 19 (1978), 25-52.

[7] F. Hofbauer. The structure of piecewise monotone transformations. Ergod. Th. \& Dynam. Sys. 1 (1981), 135-143.

[8] M. Keane. Interval exchange transformations. Math. Z. 141 (1975), 25-31.

[9] T.-Y. Li \& J. A. York. Period three implies chaos. Amer. Math. Monthly 82 (1975), 985-992.

[10] R. M. May. Biological populations obeying difference equations. $J$. Theoret. Biol. 51 (1975), 511-524.

[11] R. M. May. Simple mathematical models with very complicated dynamics. Nature 261 (1976), 459-467.

[12] Z. Nitecki. Topological dynamics on the interval. In Ergodic Theory and Dynamical Systems, II. Proc., Special Year Maryland 1979-80. A. Katok, ed., Progress in Math., Vol. 21, Birkhäuser: Boston, 1982.

[13] W. Parry. Symbolic dynamics and transformations of the unit interval. Trans. Amer. Math. Soc. 122 (1966), 368-378.

[14] C. Preston. Iterates of Maps on an Interval. Lecture Notes in Mathematics, Vol. 999, Springer: Berlin, 1983.

[15] C. Preston. Iterates of piecewise monotone maps on an interval. Preprint, Bielefeld, January 1984.

[16] C. Preston, Iterates of piecewise monotone maps on an interval. Preprint, Bielefeld, 1986.

[17] A. Rényi. Representations for real numbers and their ergodic properties. Acta. Math. Acad. Sci. Hungar. 8 (1957), 477-493.

[18] P. Walters, Ergodic Theory - Introductory Lectures. Lecture Notes in Mathematics, Vol. 458, Springer: Berlin (1975).

[19] R. F. Williams. The structure of Lorenz attractors. Publ. Math. I.H.E.S. 50 (1979), 73-99. 